\title{
Numerical treatment of the resistance term in upwind schemes in debris flow runout modelling
}

\author{
Guillermo Sánchez Burillo \\ Researcher. Dept. Suelo y Agua. Estación Experimental de Aula Dei. EEAD-CSIC. \\ P.O. Box. 202, 50080 Zaragoza, Spain.E-mail: guillermo.sanchez@csic.es \\ Santiago Beguería \\ Researcher. Dept. Suelo y Agua. Estación Experimental de Aula Dei. EEAD-CSIC. \\ P.O. Box. 202, 50080 Zaragoza, Spain. E-mail: santiago.begueria@csic.es \\ Borja Latorre \\ Researcher. Dept. Suelo y Agua. Estación Experimental de Aula Dei. EEAD-CSIC. \\ P.O. Box. 202, 50080 Zaragoza, Spain. E-mail: borja.latorre@csic.es \\ Javier Burguete. \\ Researcher. Dept. Suelo y Agua. Estación Experimental de Aula Dei. EEAD-CSIC. \\ P.O. Box. 202, 50080 Zaragoza, Spain. \\ Associated researcher. BIFI, Universidad de Zaragoza. Zaragoza, Spain. \\ E-mail: jburguete@eead.csic.es
}

\section{ABSTRACT}

Fast flows and avalanches of rock and debris are among the most dangerous of all landslide processes. Understanding and predicting post-failure motion (runout) of this kind of flowlike landslides is thus key for risk assessment, justifying the development of numerical models able to simulate their dynamics. In this work a numerical method for the resolution of the depth-averaged debris flow model is presented. This set of non-linear differential equations is formed by a variation of the shallow water equations, including strong bed slope, and a rheology resistance term. This paper focus on the numerical discretization of the resistance term, exploring three different approximations: pointwise, implicit and unified. Well-balance 
between numerical flux and source terms is only achieved using the unified discretization. In order to avoid non-physical values of the water depth and discharge, a limitation of the unified resistance term is also needed. This correction is made following three conditions that identify the physical boundaries of the resistance term in the debris flow. This technique does not affect the computational efficiency of the method, keeping the original time-step. Furthermore, proposed analytical test cases show that the three resistance limitations do not significantly perturb the numerical solution. The properties of the resulting numerical scheme are studied using a set of numerical experiments that include steady and transient flows. The results show the convenience of the unified discretization and the need of the three-condition limitation in order to avoid unphysical solutions.

Keywords: Debris flow, Shallow water, Voellmy rheology, Upwind scheme, Strong slope, Numerical friction treatment, Well-balanced scheme, Depth-averaged model, Friction physical limitation

\section{INTRODUCTION}

Rock avalanches and debris flows are flow-like landslides characterized by fast motion and high damaging potential. They constitute an important hazard in mountainous areas of the world, being responsible for loss of live and property every year. As such, significant effort has been devoted to understanding their behaviour and dynamics (Iverson 1997; Coussot and Ancey 1999). Numerical runout models have been developed to simulate the motion of granular avalanches and flows. These models are able to describe flow characteristics such as their velocity, depth and final travel length, which are of high interest for risk assessment. A number of authors developed simulation models based on the 'equivalent fluid' concept of Hungr (1995), i.e. on the assumption that the major properties of the moving mass (depth and velocity) are well described by a homogeneous fluid characterized by a rheological law (Koerner 1976; McLellan and Kaiser 1984; Kent and Hungr 1995; Hungr and Evans 1996; Rickenmann and Koch 1997; Bertolo and Wieczorek 2005; Beguería et al. 2009a). Despite the simplification involved in the equivalent fluid approach, these models proved ad- 
equate at simulating the main characteristics of laboratory and real world flows, and some of them such as DAN (Hungr 1995) and FLO-2D (O'Brien and Julien 1993) are well known by the debris flow hazard research community and have been used in a number of practical studies.

On this article we present a debris flow runout model based on the shallow water equations and the Voellmy rheological law that has been often applied to the simulation of debris flows and rock avalanches (Beguería et al. 2009b). We then discuss several issues arising from the numerical treatment of the resistance term. Three numerical correction procedures are presented, and their performance is discussed with the help of a set of case tests with and without analytical solution.

This work is focused in the numerical treatment of the resistance term in the Voellmy rheology. Some other resistance models have been proposed. For instance Takahashi (1991) suggests a model based on the collisional dilatant fluid hypothesis. Bingham model (Coussot 1997) describes laminar processes for visco-plastic materials. These are well modeled by the Coulomb rheological law (Beguería et al. 2009a) when a basal friction angle is included.

This paper is organized as follows. In the following section we present the Debris Flow Equations and introduce the strong slope model and the Voellmy rheology. Then in section "The Numerical Method: First Order Upwind Explicit Scheme" the numerical scheme is developed with detail. Next section is "Numerical Treatments and Corrections of the Resistance Term". There the numerical treatments are introduced: Unified Resistance Treatment (URT), Pointwise Resistance Treatment (PRT) and Implicit Resistance Treatment (IRT). In the same section three corrections are discussed. These are the Maximum Resistance at each Edge Limitation (MREL), the Depth-Change Limitation (DCL) and the Discharge Sign Conservation (DSC). The numerical treatments and corrections are discussed in the section "Results", where analytical and ideal dambreak tests show that just URT treatment is able to compute resistance accurately, although it is only well stabilized if the three corrections presented before are enabled at the same time. Finally, in "Conclusions" the results are 
summarized and we discuss the main conclusions of this work.

\section{DEBRIS FLOW EQUATIONS}

Assuming that a system is well described by the fluid mass and momentum conservation equations and negligible variations in the vertical coordinate, such fluid mass and momentum equations can be integrated over depth. Then, the shallow water equations are obtained. Usually debris flow and avalanche processes have been modeled with the shallow water equations by a number of authors (Laigle and Coussot 1997; Brufau et al. 2000; Denlinger and Iverson 2001; Mangeney-Castelnau et al. 2005; Beguería et al. 2009a).

In this section we present the set of equations that describes the dynamics of the system. We work with a particular realisation of the shallow water equations adapted to the description of debris flow. Debris flow equations differ from standard shallow water equations in two aspects: friction is described by a different rheology and bed slopes are usually stronger than those found in traditional shallow water problems.

\section{Strong slope pressure model}

Here we work out the pressure term in an incompressible, steady, well developed flow over a strong constant slope. Our reference frame is choosed in such a way that the system is invariant under $y$ translations, where $y=y^{\prime}$ is the axis perpendicular to both $x$ and $z$ (see fig. 1). In this case the Navier-Stokes equations are expressed as:

$$
\frac{\partial u^{\prime}}{\partial x^{\prime}}+\frac{\partial w^{\prime}}{\partial z^{\prime}}=0
$$

$$
u^{\prime} \frac{\partial u^{\prime}}{\partial x^{\prime}}+w^{\prime} \frac{\partial u^{\prime}}{\partial z^{\prime}}=g \sin \theta-\frac{1}{\rho} \frac{\partial P}{\partial x^{\prime}}+\frac{\partial}{\partial x^{\prime}}\left(\nu \frac{\partial u^{\prime}}{\partial x^{\prime}}\right)+\frac{\partial}{\partial z^{\prime}}\left(\nu \frac{\partial u^{\prime}}{\partial z^{\prime}}\right),
$$

$$
u^{\prime} \frac{\partial w^{\prime}}{\partial x^{\prime}}+w^{\prime} \frac{\partial w^{\prime}}{\partial z^{\prime}}=-g \cos \theta-\frac{1}{\rho} \frac{\partial P}{\partial z^{\prime}}+\frac{\partial}{\partial x^{\prime}}\left(\nu \frac{\partial w^{\prime}}{\partial x^{\prime}}\right)+\frac{\partial}{\partial z^{\prime}}\left(\nu \frac{\partial w^{\prime}}{\partial z^{\prime}}\right) .
$$

Observe that the equations are written in the $\left(x^{\prime}, z^{\prime}\right)$ orthogonal coordinate system, where $z^{\prime}$ is normal to the bed surface, as shown in fig. 1. For a vector velocity $\vec{v}$, the $x^{\prime}$ and $z^{\prime}$ components are $u^{\prime}$ and $w^{\prime}$, respectively. $\vec{g}$ is the gravity acceleration, $P$ the pressure, $\nu$ 
the kinematic viscosity, $\rho$ the fluid density and $\theta$ the angle of the bed with respect to the horizontal.

In the conditions depicted in fig. 1, $w^{\prime} \simeq 0$. In addition, derivatives with $x^{\prime}$ vanish. Then, system of equations (11) yields (Landau and Lifchitz 1988):

$$
g \sin \theta+\frac{\partial}{\partial z^{\prime}}\left(\nu \frac{\partial u^{\prime}}{\partial z^{\prime}}\right)=0, \quad-g \cos \theta-\frac{1}{\rho} \frac{\partial P}{\partial z^{\prime}}=0
$$

Integrating the last equation and setting $P=P_{\text {at }}$ as the atmospheric pressure in the free surface (Landau and Lifchitz 1988):

$$
P=P_{a t}+\rho g \cos \theta\left(h^{\prime}-z^{\prime}\right)
$$

where $h^{\prime}$ is the local depth in the $\left(x^{\prime}, z^{\prime}\right)$ coordinate system.

We rotate the system of equations to the Cartesian one, where $z$ is parallel to $\vec{g}$. Then, see fig. 1, $h^{\prime}=\left(z_{s}-z_{b}\right) \cos \theta$ and $z^{\prime}=\left(z-z_{b}\right) \cos \theta$ (Burguete 2003)

$$
P=P_{a t}+\rho g \cos ^{2} \theta\left(z_{s}-z\right),
$$

where in such coordinate system $z_{b}$ is the bed surface while $z_{s}$ is the free surface. In conditions of soft slopes $(\theta \approx 0, \cos \theta \approx 1)$ hydrostatic pressure is obtained:

$$
P=P_{a t}+\rho g\left(z_{s}-z\right),
$$

Note that eq. (44) corrects the hydrostatic pressure model by a $\cos ^{2} \theta$ factor.

\section{Debris flow equations}

Debris flow dynamics is often described by the so-called shallow water equations (de Saint-Venant 1871; Beguería et al. 2009a). They derive from these of Navier-Stokes, under the assumption of hydrostatic pressure and averaging over the vertical coordinate, i.e., integrating over the depth 
variable. The relationship between Navier-Stokes and shallow water equations is detailed in Burguete (2003) for several pressure models. Debris flow equations are:

$$
\frac{\partial \vec{U}}{\partial t}+\frac{\partial \vec{F}^{0}}{\partial x}=\vec{S}^{0}
$$

$$
\vec{U}=\left(\begin{array}{c}
h \\
q
\end{array}\right), \quad \vec{F}^{0}=\left(\begin{array}{c}
q \\
\frac{q^{2}}{h}
\end{array}\right), \quad \vec{S}^{0}=\left(\begin{array}{c}
0 \\
\bar{g} h\left[-\frac{\partial h}{\partial x}+S_{x}-S_{f}\right]
\end{array}\right)
$$

where we split the equation terms into: conserved variables $(\vec{U})$, quasi-conservative flux $\left(\vec{F}^{0}\right)$ and sources $\left(\vec{S}^{0}\right)$. In eq. (6) $h$ is the local depth, $q=h u$ is the discharge per unit width, $u$ is the $x$ component of the depth averaged velocity, $S_{x}=\tan \theta$ is the bed slope and $S_{f}$ is the resistance slope. Here we define $\bar{g}=g \cos ^{2} \theta$ to account for the slope angle $\theta$, as inferred in (4), and resistance to flow is described by the Voellmy rheology. Free surface friction term is assumed to be negligible. This system of equations is written in the so called quasi-conservative form.

Some numerical methods such as Finite Volume types are developed on the basis of the conservative form. In this way, these numerical algorithms force the conservation of the total flux. Observe that the system keeps invariant if we arrange its terms in the denominated conservative form:

$$
\begin{gathered}
\frac{\partial \vec{U}}{\partial t}+\frac{\partial \vec{F}^{1}}{\partial x}=\vec{S}^{1}, \\
\vec{U}=\left(\begin{array}{c}
h \\
q
\end{array}\right), \quad \vec{F}^{1}=\left(\begin{array}{c}
q \\
\frac{q^{2}}{h}+\frac{1}{2} \bar{g} h^{2}
\end{array}\right), \quad \vec{S}^{1}=\left(\begin{array}{c}
0 \\
\bar{g} h\left[S_{x}-S_{f}\right]
\end{array}\right),
\end{gathered}
$$

with $\vec{F}^{1}$ the conservative flux and $\vec{S}^{1}$ the conservative source term.

Both formulations are equivalent and produce the same solutions. We prefer the quasiconservative form because the system of equations gets slightly simplified. 


\section{Resistance model}

Several hazardous landslide processes that take place in nature are rather well described by the so called Voellmy rheology (Voellmy 1955). The Voellmy model was originally aimed at describing shear stress at the base of the flow for snow avalanches, but it has been successfully applied to granular flows of rock and debris (Koerner 1976; ; McLellan and Kaiser 1984; Kent and Hungr 1995; Hungr and Evans 1996; Rickenmann and Koch 1997; Bertolo and Wieczorek 2005) It consists of two-terms $\left(S_{f}\right)$ reaction of the bed: the basal friction and the velocity dependent (turbulent) term:

$$
S_{f}=\left(\tan \varphi+\xi \frac{q^{2}}{h^{3}}\right) \frac{q}{|q|},
$$

where $\varphi$ is the equilibrium slope angle and $\xi$ is the dynamic parameter of the resistance. The sign of $S_{f}$ is always that of $q$. When $q=0$ there is still friction. $S_{f}$ can take non-zero values, if needed, to keep (free surface) slopes smaller than the equilibrium slope.

Being relatively simple since it relies in only two empirical parameters ( $\varphi$ and $\xi)$, the Voellmy model is a convenient choice for calibration / back analysis applications.

\section{THE NUMERICAL METHOD: FIRST ORDER UPWIND EXPLICIT SCHEME}

Assuming that advection is the dominant term in the dynamics of our system, it can be classified (and numerically dealt with) as belonging to the family of hyperbolic equations. Here we define the Jacobian matrix $\mathbf{J}$ of the flux term $\vec{F}^{1}$ :

$$
\mathbf{J}=\frac{\partial \vec{F}^{1}}{\partial \vec{U}}=\left(\begin{array}{cc}
0 & 1 \\
\bar{g} h-\frac{q^{2}}{h^{2}} & 2 \frac{q}{h}
\end{array}\right) \equiv\left(\begin{array}{cc}
0 & 1 \\
c^{2}-u^{2} & 2 u
\end{array}\right)
$$

with $c=\sqrt{\bar{g} h}$ the velocity of the infinitesimal waves. So that we rewrite eq. (77) in the non-conservative form:

$$
\frac{\partial \vec{U}}{\partial t}+\mathbf{J} \frac{\partial \vec{U}}{\partial x}=\vec{S}^{1}
$$

The Jacobian has been defined with the aim of "linearizing" our equations system. Then, 
we compute the eigenvalues $\left(\lambda_{i}\right)$ and eigenvectors $\left(\vec{e}_{i}\right)$ of $\mathbf{J}$.

$$
|\mathbf{J}-\lambda I|=0 \Longrightarrow\left|\begin{array}{cc}
-\lambda & 1 \\
c^{2}-u^{2} & 2 u-\lambda
\end{array}\right|=0 \Longrightarrow \quad\left\{\begin{array}{l}
\lambda_{1}=u+c, \\
\lambda_{2}=u-c .
\end{array}\right.
$$

Then, eigenvectors are calculated:

$$
\vec{e}_{i}=\left(\begin{array}{c}
1 \\
\lambda_{i}
\end{array}\right)
$$

The Jacobian can be diagonalized as a product of matrices calculated with the eigenvalues and eigenvectors:

$$
\mathbf{J}=\mathbf{P} \mathbf{\Lambda} \mathbf{P}^{-1}, \quad \mathbf{\Lambda}=\mathbf{P}^{-1} \mathbf{J P}
$$

where

$$
\mathbf{P}=\left(\begin{array}{cc}
1 & 1 \\
\lambda_{1} & \lambda_{2}
\end{array}\right), \quad \mathbf{P}^{-1}=\frac{1}{\lambda_{2}-\lambda_{1}}\left(\begin{array}{cc}
\lambda_{2} & -1 \\
-\lambda_{1} & 1
\end{array}\right), \quad \boldsymbol{\Lambda}=\left(\begin{array}{cc}
\lambda_{1} & 0 \\
0 & \lambda_{2}
\end{array}\right)
$$

This formulation allows us to rewrite equation (10):

$$
\mathbf{P}^{-1} \frac{\partial \vec{U}}{\partial t}=\mathbf{P}^{-1}\left(\vec{S}^{1}-\mathbf{P} \boldsymbol{\Lambda} \mathbf{P}^{-1} \frac{\partial \vec{U}}{\partial x}\right)=\mathbf{P}^{-1} \vec{S}^{1}-\Lambda \mathbf{P}^{-1} \frac{\partial \vec{U}}{\partial x}
$$

Here we define a new differential variable $d \vec{\omega}=\left(\begin{array}{l}d \omega_{1} \\ d \omega_{2}\end{array}\right)$ in such a way that:

$$
\begin{gathered}
\frac{\partial \vec{\omega}}{\partial t}=\mathbf{P}^{-1} \frac{\partial \vec{U}}{\partial t}, \quad \frac{\partial \vec{\omega}}{\partial x}=\mathbf{P}^{-1} \frac{\partial \vec{U}}{\partial x} \Longrightarrow \\
\frac{\partial \vec{\omega}}{\partial t}=\mathbf{P}^{-1} \vec{S}^{1}-\Lambda \frac{\partial \vec{\omega}}{\partial x}=\vec{S}^{\prime}-\Lambda \frac{\partial \vec{\omega}}{\partial x}
\end{gathered}
$$

with $\vec{S}^{\prime}=\mathbf{P}^{-1} \vec{S}^{1}$, so the elements of $d \vec{\omega}$ are computed straightforwardly:

$$
\frac{\partial \vec{\omega}}{\partial t}=\left(\begin{array}{c}
\frac{\partial \omega_{1}}{\partial t} \\
\frac{\partial \omega_{2}}{\partial t}
\end{array}\right)=\left(\begin{array}{c}
s_{1}^{\prime}-\lambda_{1} \frac{\partial \omega_{1}}{\partial x} \\
s_{2}^{\prime}-\lambda_{2} \frac{\partial \omega_{2}}{\partial x}
\end{array}\right)
$$


In order to capture correctly the influence region, for positive $\lambda_{k}$, the evolution in $i$-th cell has to be computed considering the flux in the left wall. Similarly, negative fluxes $\left(\lambda_{k}\right)$ allow us to define the flux in the right wall:

$$
\left.\frac{\partial \omega_{k}}{\partial t}\right|_{i} ^{n}= \begin{cases}\left(s_{k}^{\prime}-\lambda_{k} \frac{\partial \omega_{k}}{\partial x}\right)_{i-1 / 2}^{n}, & \text { if } \lambda_{k} \geq 0 \\ \left(s_{k}^{\prime}-\lambda_{k} \frac{\partial \omega_{k}}{\partial x}\right)_{i+1 / 2}^{n}, & \text { if } \lambda_{k} \leq 0\end{cases}
$$

where sub-indexes $i+1 / 2$ and $i-1 / 2$ indicate, respectively, evaluation in the right and left walls of $i$-th cell. In compact notation, we write the evolution using:

$$
o_{k}^{ \pm}=\frac{1}{2}\left[1 \pm \operatorname{sign}\left(\lambda_{\mathrm{k}}\right)\right]
$$

$$
\left.\frac{\partial \omega_{k}}{\partial t}\right|_{i} ^{n}=\left[o^{+}\left(s_{k}^{\prime}-\lambda_{k} \frac{\partial \omega_{k}}{\partial x}\right)\right]_{i-1 / 2}^{n}+\left[o^{-}\left(s_{k}^{\prime}-\lambda_{k} \frac{\partial \omega_{k}}{\partial x}\right)\right]_{i+1 / 2}^{n} .
$$

At this point it is useful to define the matrices $\mathbf{O}^{ \pm}$and $\Omega^{ \pm}$:

$$
\mathbf{O}^{ \pm}=\left(\begin{array}{cc}
o_{1}^{ \pm} & 0 \\
0 & o_{2}^{ \pm}
\end{array}\right)
$$

$$
\mathbf{\Omega}^{ \pm}=\mathbf{P O}^{ \pm} \mathbf{P}^{-1}=\frac{1}{\lambda_{2}-\lambda_{1}}\left(\begin{array}{cc}
o_{1}^{ \pm} \lambda_{2}-o_{2}^{ \pm} \lambda_{1} & -o_{1}^{ \pm}+o_{2}^{ \pm} \\
\left(o_{1}^{ \pm}-o_{2}^{ \pm}\right) \lambda_{1} \lambda_{2} & -o_{1}^{ \pm} \lambda_{1}+o_{2}^{ \pm} \lambda_{2}
\end{array}\right)
$$

These matrices and eigenvalues must be computed at each cell edge. From the numerical point of view, the most accurate choice for $u$ and $c$ is to build the eigenvalues and the matrices at the cell edges as proposed by Roe (1981):

$$
u_{i+1 / 2}=\frac{u_{i} \sqrt{h_{i}}+u_{i+1} \sqrt{h_{i+1}}}{\sqrt{h_{i}}+\sqrt{h_{i+1}}}, \quad c_{i+1 / 2}=\sqrt{\bar{g} \frac{h_{i}+h_{i+1}}{2}} .
$$

Resorting to expressions (13) and getting back to the $\vec{U}$ notation, eq. (18) can be rewritten 
as:

$$
\left(\mathbf{P}^{-1} \frac{\partial \vec{U}}{\partial t}\right)_{i}^{n}=\left[\mathbf{O}^{+}\left(\mathbf{P}^{-1} \vec{S}^{1}-\Lambda \mathbf{P}^{-1} \frac{\partial \vec{U}}{\partial x}\right)\right]_{i-1 / 2}^{n}
$$

$$
+\left[\mathbf{O}^{-}\left(\mathbf{P}^{-1} \vec{S}^{1}-\mathbf{\Lambda} \mathbf{P}^{-1} \frac{\partial \vec{U}}{\partial x}\right)\right]_{i+1 / 2}^{n},
$$

and left-multiplying by $\mathbf{P}$ :

$$
\begin{aligned}
\left.\frac{\partial \vec{U}}{\partial t}\right|_{i} ^{n} & =\left(\boldsymbol{\Omega}^{+} \vec{S}^{1}-\boldsymbol{\Omega}^{+} \mathbf{J} \frac{\partial \vec{U}}{\partial x}\right)_{i-1 / 2}^{n}+\left(\boldsymbol{\Omega}^{-} \vec{S}^{1}-\boldsymbol{\Omega}^{-} \mathbf{J} \frac{\partial \vec{U}}{\partial x}\right)_{i+1 / 2}^{n} \\
& =\left[\boldsymbol{\Omega}^{+}\left(\vec{S}^{1}-\frac{\partial \vec{F}^{1}}{\partial x}\right)\right]_{i-1 / 2}^{n}+\left[\boldsymbol{\Omega}^{-}\left(\vec{S}^{1}-\frac{\partial \vec{F}^{1}}{\partial x}\right)\right]_{i+1 / 2}^{n} \\
& =\left[\boldsymbol{\Omega}^{+}\left(\vec{S}^{0}-\frac{\partial \vec{F}^{0}}{\partial x}\right)\right]_{i-1 / 2}^{n}+\left[\boldsymbol{\Omega}^{-}\left(\vec{S}^{0}-\frac{\partial \vec{F}^{0}}{\partial x}\right)\right]_{i+1 / 2}^{n} .
\end{aligned}
$$

Now, we discretize time and write the derivatives as a quotient between increments:

$$
\Delta \vec{U}_{i}^{n} \delta x_{i}=\Delta t\left[\Omega^{+}\left(\vec{S}^{0} \delta x-\delta \vec{F}^{0}\right)\right]_{i-1 / 2}^{n}+\Delta t\left[\Omega^{-}\left(\vec{S}^{0} \delta x-\delta \vec{F}^{0}\right)\right]_{i+1 / 2}^{n}
$$

where $\delta x_{i}$ is the $i$-th cell side size and $\delta x_{i+1 / 2}$ is the distance between the $i$-th and $i+1$-th cell centers (see fig. 21). Note that $\delta f_{i+1 / 2}$ terms are the difference between $f$ at $i$-th and $i+1$-th cells. Using $S_{x}=\tan \theta=-\frac{\delta z_{b}}{\delta x}$, with $z_{b}$ the bed surface, $\vec{S}^{0} \delta x$ and $\vec{F}^{0}$ arrays are:

$$
\delta \vec{F}^{0}=\left(\begin{array}{c}
\delta q \\
\delta\left(\frac{q^{2}}{h}\right)
\end{array}\right), \quad \vec{S}^{0} \delta x=\left(\begin{array}{c}
0 \\
\bar{g} h\left(-\delta h-\delta z_{b}-S_{f} \delta x\right)
\end{array}\right)
$$

The time step is computed in such a way that oscillations in the conserved variables are not enhanced.

$$
\Delta t=\mathrm{CFL} \min _{i, k}\left(\frac{\delta x}{\left|\lambda_{k}\right|}\right)_{i+1 / 2}^{n}
$$

with CFL $<1$ the dimensionless Courant-Friedrichs-Lewy number (Courant et al. 1928).

Transitions from subcritical to supercritical flow are not well resolved by the method 
described in expression (23), as shown in Burguete and García-Navarro (2004). In this reference, authors demonstrate that an entropy correction must be employed. To develop this correction we introduce some notation to be employed latter: the change in the conserved variables $\Delta \vec{U}_{i}^{n}$ can be split into predicted increment $\Delta \vec{U}^{P}$ (without resistance) and corrected increment $\Delta \vec{U}^{C}$ (only resistance):

$$
\vec{S}^{2} \delta x=\left(\begin{array}{c}
0 \\
\bar{g} h\left(-\delta h-\delta z_{b}\right)
\end{array}\right), \quad \vec{S}^{f} \delta x=\left(\begin{array}{c}
0 \\
-\bar{g} h S_{f} \delta x
\end{array}\right)
$$

$$
\vec{A}_{i+1 / 2}^{ \pm}=\left(\begin{array}{l}
a_{h} \\
a_{q}
\end{array}\right)_{i+1 / 2}^{ \pm}=\left[\Omega^{ \pm}\left(\vec{S}^{2} \delta x-\delta \vec{F}^{0}\right) \mp \mu \delta \vec{U}\right]_{i+1 / 2}^{n},
$$

" .

where $\mu$ is an artificial viscosity coefficient (Burguete and García-Navarro 2004) computed as follows:

$$
\mu_{i+1 / 2}=\max _{k}\left\{\begin{array}{lc}
\frac{\left(\lambda_{k}\right)_{i+1}-\left(\lambda_{k}\right)_{i}}{4}, & \text { if }\left(\lambda_{\mathrm{k}}\right)_{\mathrm{i}+1}>0 \text { and }\left(\lambda_{\mathrm{k}}\right)_{\mathrm{i}}<0 ; \\
0, & \text { otherwise }
\end{array}\right.
$$

Observe that $\mu \neq 0$ only in transitions from subcritical to supercritical flow.

\section{NUMERICAL TREATMENTS AND LIMITATIONS OF THE RESISTANCE TERM}

This section is structured as follows: First, we present three numerical treatments of the resistance term, pointwise resistance treatment, implicit resistance treatment, and unified resistance treatment. Then, we present three protection methods to deal with the unphysical effects arising from the overestimation of resistance, these are the maximum resistance at 
each edge limitation, depth-change limitation and discharge sign conservation.

\section{Numerical resistance treatments}

Pointwise resistance treatment (PRT)

It has been suggested that pointwise discretization of resistance provides numerical stable results with a lower computing effort (Brufau et al. 2000; Delis et al. 2011). That consists, essentially, in the evaluation of the resistance contribution at the center of each cell, instead of the cell edges. The numerical scheme as described in eq. (26) is modified in the following manner:

$$
\vec{B}_{i+1 / 2}^{-}=\vec{B}_{i-1 / 2}^{+}=\frac{1}{2}\left(\vec{S}^{f} \delta x\right)_{i}^{n}
$$

However, in Burguete et al. (2008) authors demonstrated that PRT does not provide a correct balance among terms of the shallow water equations.

Implicit resistance treatment (IRT)

Implicit treatment of the resistance term has been often presented as a solution for the instabilities and numerical oscillations that may appear when dealing with resistance in explicit schemes (Brufau et al. 2000; Burguete and García-Navarro 2001; Delis et al. 2011). In this case eq. (26) is modified as:

$$
\vec{B}_{i+1 / 2}^{-}=\vec{B}_{i-1 / 2}^{+}=\frac{1}{2}\left(\vec{S}^{f} \delta x\right)_{i}^{n+1}
$$

So, the IRT is implemented in the following way:

$$
q_{i}^{P}=q_{i}^{n}+\Delta q_{i}^{P}, \quad q_{i}^{n+1}=q_{i}^{P}-\Delta t(\bar{g} h)_{i}^{n+1} S_{f}\left(q_{i}^{n+1}, h_{i}^{n+1}\right) .
$$

For several resistance models, as the one we are working with, it is possible to work out the value of $q_{i}^{n+1}$. Let us introduce the resistance expression (8) into the implicit scheme (301). Thus,

$$
q_{i}^{n+1}=q_{i}^{P}-\Delta t(\bar{g} h)_{i}^{n+1}\left(f\left(h_{i}^{n+1}\right)\left(q_{i}^{n+1}\right)^{2}+\tan \varphi\right) \frac{q_{i}^{P}}{\left|q_{i}^{P}\right|}
$$


where the dependence of $S_{f}$ with $h$ has been enclosed in $f(h)=\frac{\xi}{h^{3}}$. For the moment, realisation of $f(h)$ either in $t^{n}$ or $t^{n+1}$ is irrelevant for us. Observe that $f(h)$ is defined positive. In practice, the sign of the resistance contribution (i.e. $q_{i}^{n+1}$ ) is given by $q_{i}^{P}$. Otherwise, there would be a sign indetermination when $q=0$.

Expression (31) includes two equations that must be solved separately for positive and negative $q_{i}^{P}$. They are two second order equations, each one of them with two mathematical solutions. Here negative root must be discarded since it entails a change in the sign of $q$ : it implies different senses for the discharge with and without resistance, which is an undesired numerical overestimation of resistance. Then, the solution for $q_{i}^{n+1}$ can be written using a general expression for positive and negative values of $q_{i}^{P}$ :

$$
q_{i}^{n+1}=\frac{-1+\sqrt{1-4 \Delta t(\bar{g} h)_{i}^{n+1} f\left(h_{i}^{n+1}\right)(\Delta t \bar{g} h \tan \varphi-|q|)_{i}^{n+1}}}{2 \Delta t(\bar{g} h)_{i}^{n+1} f\left(h_{i}^{n+1}\right)} \frac{q_{i}^{P}}{|q|_{i}^{P}}
$$

Physically, resistance can slow down the moving mass, but never change the sense of motion. The choice of the right root does not imply necessarily sign conservation. The squared root needs to be greater than 1 (remind that $\bar{g} h f(h)>0$ ). This introduces a time step restriction:

$$
1-4 \Delta t(\bar{g} h)_{i}^{n+1} f\left(h_{i}^{n+1}\right)(\Delta t \bar{g} h \tan \varphi-|q|)_{i}^{n+1}>1 \Longrightarrow
$$

$$
\Delta t<\frac{|q|_{i}^{P}}{(\bar{g} h)_{i}^{n+1} \tan \varphi} .
$$

Thus, the IRT does not fulfill the stability criterion of no-sign-change condition for Voellmy rheologies: if $q \rightarrow 0, \Delta t \rightarrow 0$. 


\section{Unified resistance treatment (URT)}

First order upwind method as described in eqs. (23) and (26) requires the evaluation of the resistance term at the cell walls, as well as any other term of the equations.

$$
h_{i+1 / 2}=\frac{h_{i}+h_{i+1}}{2}, \quad q_{i+1 / 2}=\frac{q_{i}+q_{i+1}}{2}, \quad\left(S_{f}\right)_{i+1 / 2}=S_{f}\left(h_{i+1 / 2}, q_{i+1 / 2}\right) .
$$

We denominate this as unified resistance treatment (URT).

Burguete et al. (2008) shows that URT provides a well-balanced scheme for shallow water steady flows.

\section{Limitations to the numerical resistance}

Because of its numerical realisation, calculated resistance inside some cell might be larger than the physically maximum allowed resistance, i.e., the value such that flow is stopped. This overestimation of resistance is due to two main reasons: numerical integration of the resistance term is not exact and upwind schemes may introduce non-physical effects in the mass conservation equation. It is important to stress that the flow equations (6) with the Voellmy rheology does not produce unphysical effects, while the numerical treatment of the $S_{f}$ contribution is the only source of such kind of errors. Under no resistance conditions, the upwind scheme (with the CFL restriction) produces adequate solutions.

Observe that our rheology equation states that, as the absolute value of the discharge decreases $|q| \rightarrow 0$, the resistance level tends asymptotically to its minimum value $\left|S_{f}\right| \rightarrow$ $\tan \varphi$. For small $q$ and small $\delta q, \delta z_{b}$ and $\delta h$, the resistance contribution to the evolution of the conserved variables in some cell may be dominand: $B_{i \pm 1 / 2}^{ \pm}$terms are dominant over $A_{i \pm 1 / 2}^{ \pm}$terms in eq. (26). As a consequence, numerical errors may appear and, eventually, propagate. For instance, there may be cases such as the one described in fig. 3: very small depth differences and equal non-zero discharges over horizontal bed, such that the only flux terms across the cell wall are the -small- source because of pressure differences and the -largeresistance. 
In the hypothetic case where $S_{f}=0$, a time step later the difference $d h$ between cells $i-1$ and $i$ should decrease. However, a large resistance response may deal to increases in $d h$, which is physically senseless.

Similarly, unphysical results of $q$ may be obtained. For instance, the sign of $q$ with and without resistance might be different, while it is well known that resistance can stop the moving mass, but it is unable to change the sense of motion. In this section we discuss several techniques to fix the resistance term. Some techniques to stabilize resistance are: time step reduction (Murillo et al. 2007; Beguería et al. 2009a) or grid characteristic distance reduction (Burguete et al. 2007). In Murillo et al. (2009), URT is preferently employed, and replaced by the more stable (but less accurate) PRT method in those cells where stability problems arise. A set of resistance fixes is proposed in Murillo and García-Navarro (2012) to avoid negative depth solutions produced by numerical overestimation of resistance effect. In this paper we present a set of techniques to deal with Voellmy resistance in such a way that no limitations in time step size, cell size or accuracy are introduced.

Maximum resistance at each edge limitation (MREL)

The contribution of the resistance to the evolution of the discharge is analyzed in (Burguete et al. 2007; Burguete et al. 2008). Let us take the $i+1 / 2$-th edge defined by $i$-th and $i+1$-th cells, as shown in fig. 4. Integrating the quasi-conservative equation (6) between $x_{i}$ and $x_{i+1}$ :

$$
\int_{t^{n}}^{t^{n+1}} d t \int_{x_{i}}^{x_{i+1}} d x\left(\frac{\partial \vec{U}}{\partial t}+\frac{\partial \vec{F}^{0}}{\partial x}-\vec{S}^{0}\right)=\overrightarrow{0}
$$

this equation can be approximated as:

$$
\frac{\vec{U}_{i}^{n+1}+\vec{U}_{i+1}^{n+1}-\vec{U}_{i}^{n}-\vec{U}_{i+1}^{n}}{2} \delta x_{i+1 / 2}+\Delta t\left[\left(\vec{F}^{0}\right)_{i+1}^{n}-\left(\vec{F}^{0}\right)_{i}^{n}-\left(\vec{S}^{0} \delta x\right)_{i+1 / 2}^{n}\right]=\overrightarrow{0}
$$

The second component of this vectorial equation can be split into predicted and corrected terms. By predicted term we mean the result for $q$ that would be obtained under the hypothetical condition of no resistance. The corrected contribution is obtained if only $S_{f}$ is 
introduced in equation (6)

$$
\begin{gathered}
q_{i+1 / 2}^{n+1}=q_{i+1 / 2}^{P}-\left(T_{f}\right)_{i+1 / 2}^{n}, \\
q_{i+1 / 2}^{P}=\frac{q_{i}^{n}+q_{i+1}^{n}}{2}-\Delta t\left[\bar{g} h\left(\delta h+\delta z_{b}\right)+\delta\left(\frac{q^{2}}{h}\right)\right]_{i+1 / 2}^{n} \\
\left(T_{f}\right)_{i+1 / 2}^{n}=\Delta t\left(\bar{g} h \delta x S_{f}\right)_{i+1 / 2}^{n} .
\end{gathered}
$$

According to Burguete et al. (2008), to avoid unphysical changes of $q$ sign, the corrector contribution to the variation of $q$ across each edge should not be larger than $q^{P}$ ( $\Longrightarrow\left|T_{f}\right| \leq$ $\left.q^{P}\right)$. It is achieved making:

$$
\begin{gathered}
q_{i+1 / 2}^{n+1}=q_{i+1 / 2}^{P}-\left(T_{f}\right)_{i+1 / 2}^{P}, \\
\left(T_{f}\right)_{i+1 / 2}^{P}= \begin{cases}\left(T_{f}\right)_{i+1 / 2}^{n}, & \text { if }\left|T_{f}\right|_{i+1 / 2}^{n} \leq|q|_{i+1 / 2}^{P} \\
|q|_{i+1 / 2}^{P}\left(\frac{T_{f}}{\left|T_{f}\right|}\right)_{i+1 / 2}^{n}, & \text { if }\left|T_{f}\right|_{i+1 / 2}^{n}>|q|_{i+1 / 2}^{P}\end{cases}
\end{gathered}
$$

Equation (38) is the basis of the MREL method and it provides, for every single cell edge, a maximum value of $T_{f}$ which should not be exceeded.

Depth-change limitation (DCL)

Another undesired effect of the inaccurate treatment of the resistance term is the excessive reaction in depth. Despite the resistance reaction vector $\vec{S}^{f}$ is not supposed to change depth, in the URT method a correcting term in $h$ may arise because of the the matrix decomposition of $\vec{B}^{ \pm}$in eq. (26). Then, with URT method, although the $h$ component is 0 in $\vec{S}^{f}$, in $b_{h}^{ \pm}$it is not, in general.

The numerical scheme does not insure that the predicted increment in $a_{h}^{ \pm}$is larger than the correcting term $b_{h}^{ \pm}$. Furthermore, the URT method often produces situations where $\left|b_{h}^{ \pm}\right|>\left|a_{h}^{ \pm}\right|$while $b_{h}^{ \pm} \cdot a_{h}^{ \pm}<0$. This is contradictory with the idea of resistance as a passive mitigation of the result of the evolution of the conserved variables but not as an active counteracting contribution larger than any other.

Here we propose a new correction to the resistance contribution which consists on the reduction (of the absolute value) of the resistance term $b_{h}^{ \pm}$when it is too large. 
Note that this must be done carefully to keep the mass conservation. In this protection we reduce the correcting increment in $b_{h}^{ \pm}$, when its absolute value is larger than that of the predicting increment $a_{h}^{ \pm}$. Since our numerical method provides two correcting terms evaluated in the edge between cells $i$ and $i+1$, we must check in both components either if there is a surplus $(S P)$ or not:

$$
S P_{i+1 / 2}=\max \left(\frac{\left|b_{h}\right|_{i+1 / 2}^{+}-\left|a_{h}\right|_{i+1 / 2}^{+}}{\delta x_{i+1}}, \frac{\left|b_{h}\right|_{i+1 / 2}^{-}-\left|a_{h}\right|_{i+1 / 2}^{-}}{\delta x_{i}}, 0\right)
$$

Then, this surplus or excessive resistance contribution to $h$ must be taken out. This is done by modifying the balance between cells $i$ and $i+1$.

$$
\begin{aligned}
& \left(b_{h}^{\prime}\right)_{i+1 / 2}^{+}=\left(b_{h}\right)_{i+1 / 2}^{+}-S P_{i+1 / 2} \frac{\left(b_{h}\right)_{i+1 / 2}^{+}}{\left|b_{h}\right|_{i+1 / 2}^{+}} \delta x_{i+1}, \\
& \left(b_{h}^{\prime}\right)_{i+1 / 2}^{-}=\left(b_{h}\right)_{i+1 / 2}^{-}+S P_{i+1 / 2} \frac{\left(b_{h}\right)_{i+1 / 2}^{+}}{\left|b_{h}\right|_{i+1 / 2}^{+}} \delta x_{i},
\end{aligned}
$$

where the vector $\Delta \vec{U}^{C}$ in eq. (26) is substituted by:

$$
\left(\vec{B}^{\prime}\right)^{ \pm}=\left(\begin{array}{c}
b_{h}^{\prime} \\
b_{q}
\end{array}\right)^{ \pm}, \quad \Delta \vec{U}_{i}^{C}=\frac{\Delta t}{\delta x_{i}}\left[\left(\vec{B}^{\prime}\right)_{i+1 / 2}^{-}+\left(\vec{B}^{\prime}\right)_{i-1 / 2}^{+}\right]
$$

The excessive resistance is removed keeping the mass balance.

Note that both MREL and DCL corrections modify the numerical fluxes at the edges. Then, these methods can be only applied to the URT method.

\section{Discharge sign conservation (DSC)}

MREL provides (when needed) a reduction in the effective corrector term for each cell edge. The aim of MREL is to keep the sign of the predicted $q$. To do so, the method takes into account the predicted $q$ flow across each cell edge.

However, the sum of all edges contribution to the dynamics in a single cell might not 
preserve the $q$-sign, even if MREL applies. Indeed, after accounting for each edge predictor and then each edge corrector term contributions, the method might produce in some cells predicted and corrected $q$ values with different signs.

To solve such undesired solutions for $q$, we propose a new scheme. Resistance is treated separately from other contributions. The total predicted variation (i.e., accounting for each edge) of the conserved variables $\Delta \vec{U}_{i}^{P}$ is computed with $S_{f}=0$, and a corrector term $\Delta \vec{U}_{i}^{C}$ is computed with only resistance. At every single cell, one must update $\vec{U}_{i}$ performing two steps between time step $t^{n}$ and $t^{n+1}=t^{n}+\Delta t$ :

$$
\vec{U}_{i}^{P}=\vec{U}_{i}^{n}+\Delta \vec{U}_{i}^{P}, \quad \vec{U}_{i}^{C}=\vec{U}_{i}^{P}+\Delta \vec{U}_{i}^{C}
$$

The second component of $\vec{U}$, the discharge $q$ might have different signs in its predicted and corrected realisations. This two steps scheme corrects this undesired solution.

$$
h_{i}^{n+1}=h_{i}^{C}, \quad q_{i}^{n+1}=\left\{\begin{array}{lc}
0, & \text { if } q_{i}^{P} \cdot q_{i}^{C}<0 \\
q_{i}^{C}, & \text { otherwise. }
\end{array}\right.
$$

Observe that DSC is intuitive since the change of the sign of $q$ should be interpreted as an overestimation of the effective $S_{f}$. This term may stop $q$ but never change its sign. This correction modifies the updated value of $q$ inside each cell if needed. Therefore, it can be applied to the URT, PRT and IRT methods.

DSC works on the cell, just taking into account $q$ values inside each cell. On the other hand, MREL works on cell edges.

\section{RESULTS}

In this section we present several test cases, some of which are with analytical result, in order to evaluate the methods presented in the previous section.

Whenever some modification is introduced in a numerical method, it is important to check if we are distorting the solution or not. In principle, resistance protection schemes 
might dramatically change the solution. Here we present some examples with analytical solution and some ideal dambreaks to evaluate the impact of the protection methods in the resolution of the flow.

\section{Steady flow tests with analytical solution}

In Burguete et al. (2008), it was shown that pointwise resistance (see eq. (28)) may deal to a wrong balance in the shallow water equations terms. This conclusion was achieved after studying a MacDonald test case (MacDonald et al. 1997) with analytical solution. Here we follow the same procedure and propose new tests for the Voellmy resistance.

Here we proceed analogously to MacDonald et al. (1997) to obtain steady flow tests with analytical solution. In such test cases, $q$ is invariant under time-space translations and $h$ is constant in time. The balance equations are:

$$
\frac{\partial q}{\partial x}=0, \quad \frac{\partial}{\partial x}\left(\frac{q^{2}}{h}+\frac{1}{2} \bar{g} h^{2}\right)=\bar{g} h\left(S_{x}-S_{f}\right) .
$$

In the limit $S_{x}^{2}=\left(\frac{\partial z_{b}}{\partial x}\right)^{2} \ll 1, \bar{g}$ can be approximated by $g$ :

$$
\bar{g}=\frac{g}{1+\tan ^{2} \theta}=\frac{g}{1+\left(\frac{\partial z_{b}}{\partial x}\right)^{2}} \approx g .
$$

If $h(x)$ is analytic, then another analytical expression can be obtained for the bed source $S_{x}$ :

$$
S_{x}=-\frac{\partial z_{b}}{\partial x}=S_{f}+\frac{1}{g h} \frac{\partial}{\partial x}\left(\frac{q^{2}}{h}+\frac{1}{2} g h^{2}\right) .
$$

We use the following analytical $h$ :

$$
h=h_{0}+0.2 \sin \left(\frac{2 \pi x}{L}-\frac{\pi}{2}\right)
$$

where $L$ is the length of the domain. In our numerical test, $L=200 \mathrm{~m}$. Integrating numerically eq. (46), the shape of the soil bed $z_{b}(x)$ is achieved. We have performed two 
different simulations in subcritical and supercritical regimes with Voellmy rheology. In table1. the parameters of each case are shown. We have done these simulations using $\delta x=2 \mathrm{~m}$ and $\mathrm{CFL}=0.9$.

The shapes of $h$ and $z_{b}$ are depicted in fig. 5 for the subcritical case. Note that the slope is around 0.06. That confirms that $\bar{g} \approx \frac{g}{1+0.06^{2}} \approx 0.9996 g \approx g$ is a good approximation. In fig. 6 the $h$ and $q$ profiles in equilibrium are depicted for the unified method and PRT for the subcritical case. In the URT simulation, the resistance fix set made up by MREL, DCL and DSC limitations has been applied, while in the pointwise case only DSC limitation has been activated, for the reasons explained before. In fig. 7 we depict the functions $h$ and $z_{b}$ for the supercritical case. In this case, $\bar{g} \approx \frac{g}{1+0.15^{2}} \approx 0.98 g \approx g$ is a reasonable approximation. Results of $h$ and $q$ in equilibrium are plotted in fig. 8. The main conclusion is that the PRT for the Voellmy resistance works well only in supercritical flows. This is an unexpected result and further research is needed to clarify this point. In subcritical states, the balance of terms fails with this method. URT solves accurately the debris flow in both cases. The proposed corrections MREL, DSC and DCL do not perturb the steady state solutions.

\section{Ideal dambreak}

Dambreak is a classical test-bed to check the performance of hydraulic numerical simulations, since it can detect the unbalanced terms and physical inconsistencies, even if it has no analytical solution in general. By ideal dambreak we mean an initial situation such that on each side of the discontinuity, $h=$ const., and $q=0$. Here we present two cases of ideal dambreaks over a dry bed. The domain size is $L=200 \mathrm{~m}$, and the discontinuity in $h$ takes

place at $x=\frac{L}{2}$. Our study cases are characterized by the parameters shown in table 2. We have done these simulations using $\delta x=2 \mathrm{~m}$ and $\mathrm{CFL}=0.9$.

\section{Case I}

In the Case I dambreak the purely frictional rheology is studied. This case does not consider the turbulent term of the resistance. Despite we shown that IRT is not suited for our rheology (see eq. (33) $)$, we present here the results of our simulation. In fig. 9 we show 
the results.

Remind that we expected wrong results in the IRT, since there is no time step to ensure that the friction keeps the sign of the discharge. Indeed, we observe in fig. 9 that $q$ is negative in the nearby of the shock, even if we enable the DSC limitation. In addition, the local depth becomes higher than it was in the left side of the front. This is an undesired effect that does not take place when friction is computed with the URT with the limitations set (MREL+DCL+DSC), as shown in the same plot.

Keeping in the URT, the same simulation illustrates the importance of the MREL. We have simulated such system activating the following corrections: MREL (eq. (38)), DSC (eq. (43)) and DCL (eqs. (39), (40) and (41)). Then, we have disabled the MREL and compared results. After a simulated time 10 s, we observe several differences in figs. [10] and 11 .

When MREL correction is disabled, we detect roughness in the equilibrium profile of $h$. However, the profile becomes flat after enabling it. In addition, we detect sharp peaks in $q$ if MREL protection is disabled. We can conclude that the MREL correction stabilizes the resistance term when dealing with purely frictional rheology.

Case II

Here both Voellmy terms contribute to the debris flow dynamics. This case has been used to test either the DSC (see eq. (43)) needs to be enabled or not. With this particular protection one does not need to go to purely frictional rheologies in order to detect undesired effects.

In fig. 12 we observe negative values in $q$ at $t=1 \mathrm{~s}$ when the DSC is not enabled, despite MREL and DCL corrections are activated. This is due to an excessive resistance response to the flow generated by the dambreak. Observe that this undesired effect vanishes if we let our DSC protection work.

Ideal dambreak test case II has been simulated to check the DCL too. Here we show that the protection that does not permit anti-advection changes in the depth across the walls is 
necessary as well. The last example is illuminating. If the DCL protection is activated, there are no abnormal results. However, when it is disabled, we detect peaks in depth in the front of the dambreak, see fig. 13. This test shows that the DCL correction is necessary for the correct computation of the resistance in our rheology model.

\section{Invariant depth test}

Another case with analytical solution to check the performance of the resistance numerical treatment is presented here. It consists of a current over flat bed where $h$ is invariant under space-time translations and $q$ is invariant under spatial translations. Here $q$ is expected to decay in time (because of the resistance) until the flow stops. The system of equations is:

$$
\frac{\partial h}{\partial t}=\frac{\partial h}{\partial x}=\frac{\partial q}{\partial x}=\frac{\partial z_{b}}{\partial x}=0 \quad \Longrightarrow \quad \frac{\partial q}{\partial t}=-\bar{g} h S_{f}
$$

Then, we substitute expression (8) :

$$
\frac{\partial q}{\partial t}=-\bar{g} h\left(\tan \varphi+\xi \frac{q^{2}}{h^{3}}\right) \frac{q}{|q|}
$$

For simplicity we solve the $q>0$ case:

$$
t_{\text {decay }}=\sqrt{\frac{h}{\bar{g}^{2} \xi \tan \varphi}} \arctan \left(\sqrt{\frac{\xi q_{0}}{h^{3} \tan \varphi}}\right)
$$

$$
q= \begin{cases}\sqrt{\frac{h^{3} \tan \varphi}{\xi}} \tan \left[\arctan \left(\sqrt{\frac{\xi q_{0}}{h^{3} \tan \varphi}}\right)-\sqrt{\frac{\bar{g}^{2} \xi \tan \varphi}{h}} t\right], & \text { if } t<t_{\mathrm{decay}} \\ 0, & \text { if } t \geq t_{\mathrm{decay}}\end{cases}
$$

where $q_{0}$ is $q(t=0)$. To solve this problem accurately, we need to take time step sizes much smaller than the decay time $\left(\Delta t \ll t_{\text {decay }}\right)$. Observe that resistance contribution, if overestimated, may deal to $q<0$ solutions. Thus the performance of both MREL and DSC limitations is tested in this case. 
We have run a case with the following parameters:

$$
q_{0}=10 \mathrm{~m}^{2} / \mathrm{s}, \quad h=10 \mathrm{~m}, \quad \tan \varphi=0.01, \quad \xi=0.003 .
$$

We have done these simulations using $\delta x=2 \mathrm{~m}$ and $\mathrm{CFL}=0.9$. This set of parameters makes the decay non-linear, but the typical time step size is still small enough: $\Delta t \approx 0.02 \mathrm{~s}$ $\ll t_{\text {decay }} \approx 10.1 \mathrm{~s}$.

In fig. 14 we show the numerical results with and without limitations, together with the analytical decay for $q(t)$. The decay function is well reproduced by the simulation in both cases. The numerical results only differ themselves when $q \rightarrow 0$, when both DSC and MREL activate. When these limitation methods are activated, the decay is softer. We observe that the limitations do not introduce a significant change in the discharge. Furthermore, DSC and MREL avoid wrong numerical solutions such as the triangle wave-like shape of $q(t)$ when the mass is supposed to stop.

\section{Discontinuity within normal flow (DNF) test}

Burguete et al. (2006) developed some analytical tests based on the Rankine-Hugoniot equation for 1D propagating shock waves with a Gauckler-Manning rheology and normal flows. In this section we propose another analytical test, adapting the previous procedure to a Voellmy rheology.

Here we have a constant slope, $S_{x}$ and the Voellmy expression for $S_{f}$. In the fig. 15 we observe a discontinuity propagating with velocity $U$. In the left hand side of the discontinuity, there are constant velocity and discharge: $h_{1}$ and $q_{1}$. In the right hand side, $h_{2}$ and $q_{2}$ are constant as well. In both sides of the shock, the space-time derivatives of $h$ and $q$ vanish. Then, eq. (7) becomes:

$$
\begin{gathered}
\bar{g} h\left(S_{x}-S_{f}\right)=0 \Longrightarrow \\
S_{x}=\tan \varphi+\xi \frac{q_{1}^{2}}{h_{1}^{3}}=\tan \varphi+\xi \frac{q_{2}^{2}}{h_{2}^{3}}
\end{gathered}
$$


having a normal flow $\left(S_{x}=S_{f}\right)$ at both sides of the discontinuity. In addition, in the discontinuity limits, according to Burguete et al. (2006):

$$
\begin{aligned}
h_{1}\left(\frac{q_{1}}{h_{1}}-U\right) & =h_{2}\left(\frac{q_{2}}{h_{2}}-U\right), \\
h_{1}\left(\frac{q_{1}}{h_{1}}-U\right)^{2}+\frac{1}{2} \bar{g} h_{1}^{2} & =h_{2}\left(\frac{q_{2}}{h_{2}}-U\right)^{2}+\frac{1}{2} \bar{g} h_{2}^{2} .
\end{aligned}
$$

expressions (51) and (52) make up a four equations system with eight variables $\left(S_{x}, \varphi, \xi, q_{1}\right.$, $q_{2}, h_{1}, h_{2}$ and $\left.U\right)$. Then, there are four degrees of freedom. We set the following parameters:

$$
S_{x}=0.3, \quad \tan \varphi=0.1, \quad \xi=0.004 s^{2} / m, \quad h_{2}=0.4 m
$$

and solving the four equations system we obtain:

$$
h_{1}=1.5576 \mathrm{~m}, \quad q_{2}=1.7888 \mathrm{~m}^{2} / \mathrm{s}, \quad q_{1}=13.7463 \mathrm{~m}^{2} / \mathrm{s}, \quad U=10.3291 \mathrm{~m} / \mathrm{s} .
$$

The domain size is $L=2000 \mathrm{~m}$. We have done these simulations using $\delta x=2 \mathrm{~m}$ and $\mathrm{CFL}=0.9$.

In fig. 16 we plot the depth at time $t=0 \mathrm{~s}$ and $t=25 \mathrm{~s}$ with and without protections. Observe that protections do not change the result. In addition the velocity of the shock matches up very well with the analytical result for $U$.

\section{CONCLUSIONS}

The Voellmy rheology, as shown, deals to undesired effects whenever resistance contribution is not treated carefully. In this paper we demonstrate that two typical techniques for the stability of the resistance such as IRT and PRT are not appropriate in general for Voellmy rheologies. PRT produces wrong solutions in the subcritical steady flow test as shown in figs. 5] and 6, and non-physical solutions are obtained if resistance contribution is computed implicitly, as shown in fig. 9. Only URT is able to produce well balanced outputs, 
although some resistance fix techniques are needed to avoid undesired effects, mainly due to the numerical overestimation of resistance.

The already existing technique of MREL (see eq. (38)) has been shown to improve the results. Although necessary, this technique is insufficient to correctly solve the system. In this paper we propose two new methods to stabilize resistance and avoid unphysical solutions:

These are (i) DSC (eq. (43)), splitting the time step evolution of the discharge into predicting and correcting contributions and (ii) DCL (eqs. (39), (40) and (41)) affecting the numerical flux across cell edges. These methods have been checked with dambreak tests that demonstrate that each one of them is needed in order to produce physically acceptable solutions. In addition, they do not produce spurious effects when compared with analytical tests: the numerical solution of Steady Flow Test matches up pretty well when the set of protections is activated (see figs. 6 and 8). Besides, the physical inconsistencies in the Invariant depth simulation (see fig. 14) and the Rankine-Hugoniot discontinuity within normal flow (see fig. 16) vanish when the set of protections applies. When enabled, the protection methods only modify the solution whenever it is necessary just to avoid undesired numerical results.

Finally, two aspects about the techniques suggested in this paper should be stressed. First, our resistance fix set (URT+MREL+DSC+DCL) produces solutions that make sense, from the viewpoint of physics, not only in the steady or equilibrium state, but also in the transient states. Second, such results are obtained with CFL values close to 1, at least for the tests performed in this paper. Similarly, no time step reduction is required. Thus, we can conclude that the resistance fix presented here is efficient from the physical and computational points of view.

\section{ACKNOWLEDGEMENTS}

This research was supported by project ChangingRISKS (OPE00446 / PIM2010ECR00726) financed by EU ERA-NET CIRCLE Programme, and Grupo de Excelencia E68 financed by the Aragón Government and the European Social Fund (ESF). 
$\Delta q^{C}=$ Corrector term of $\mathrm{q}$.

$\Delta q^{P}=$ Total predicted variation of $q$ (i.e., with $S_{f}=0$ ).

$\Delta t=$ Time step

$\Delta \vec{U}^{C}=$ Total correction variation of $\vec{U}$ (i.e. with only $S_{f}$ ).

$\Delta \vec{U}_{i}^{n}=$ Variation in $\vec{U}$ at $n$-th time step in $i$-th cell.

$\Delta \vec{U}^{P}=$ Total predicted variation of $\vec{U}$ (i.e. with $\left.S_{f}=0\right)$.

$\delta x_{i}=i$-th cell size.

$\theta=$ Bed angle.

$\Lambda=$ Eigenvalues diagonal matrix.

$\lambda_{i}=i$-th eigenvalue of $\mathbf{J}$.

$\mu=$ Artificial viscosity coefficient for entropy correction.

$\nu=$ Kinematic viscosity.

$\xi=$ Dynamic parameter of resistance.

$\rho=$ Fluid density.

$\varphi=$ Equilibrium angle or static parameter of friction.

$\mathbf{\Omega}^{ \pm}=$Upwind matrices.

$\vec{A}_{i+1 / 2}^{ \pm}=$Contribution vector to $\Delta \vec{U}^{P}$ across the $i+1 / 2$-th edge.

$a_{h}^{ \pm}=$First component of $\vec{A}^{ \pm}$.

$a_{q}^{ \pm}=$Second component of $\vec{A}^{ \pm}$. 
$\vec{B}_{i+1 / 2}^{ \pm}=$Contribution vector to $\Delta \vec{U}^{C}$ across the $i+1 / 2$-th edge.

$\left(\vec{B}^{\prime}\right)_{i+1 / 2}^{ \pm}=$Corrected $\vec{B}^{ \pm}$in DCL method.

$b_{h}^{ \pm}=$First component of $\vec{B}^{ \pm}$.

$\left(b_{h}^{\prime}\right)^{ \pm}=$First component of $\left(\vec{B}^{\prime}\right)^{ \pm}$

$b_{q}^{ \pm}=$Second component of $\vec{B}^{ \pm}$and $\left(\overrightarrow{B^{\prime}}\right)^{ \pm}$

$\mathrm{CFL}=$ Courant-Friedrichs-Lewy number.

$c=$ Velocity of the infinitesimal waves $c=\sqrt{\bar{g} h}$.

$d \vec{\omega}=$ Differential characteristic variable: $d \vec{\omega}=\mathbf{P}^{-1} d \vec{U}$.

$\vec{e}_{i}=i$-th eigenvector of $\mathbf{J}$.

$\vec{F}^{0}=$ Quasiconservative flux.

$\vec{F}^{1}=$ Conservative flux.

$f(h)=h$-dependent term of $S_{f}$.

$g=$ Gravity acceleration

$\bar{g}=$ Effective gravity in developed flow: $\bar{g}=g \cos ^{2} \theta$.

$h=$ Depth

$h_{0}=$ Average depth of $h$ in the MacDonald-like tests.

$h_{1}=$ Initial $h$ in the left hand side of discontinuity in the 'DNF test'.

$h_{2}=$ Initial $h$ in the right hand side of discontinuity in the 'DNF test'.

$h_{i}=$ Initial depth in the left hand side of dambreak discontinuity.

$\mathbf{J}=$ Jacobian of $\vec{F}^{1}$. 
$L=$ System size.

$\mathrm{O}^{ \pm}=$Diagonal sign matrices.

$o_{i}^{ \pm}=i$-th diagonal term of $\mathbf{O}^{ \pm}$matrix

$P=$ Fluid pressure.

$\mathbf{P}=$ Eigenvectors of $\mathbf{J}$ matrix.

$P_{a t}=$ Atmospheric pressure.

$q=$ Discharge per unit width.

$q_{0}=$ Initial $q$ in the 'invariant depth test'.

$q_{1}=$ Initial $q$ in the left hand side of discontinuity in the 'DNF test'.

$q_{2}=$ Initial $q$ in the right hand side of discontinuity in the 'DNF test'.

$q^{C}=$ Corrected value of $q$.

$q^{P}=$ Predicted value of $q$

$\overrightarrow{S^{\prime}}=$ Auxiliary vector: $\vec{S}^{\prime}=\mathbf{P}^{-1} \vec{S}^{1}$.

$\vec{S}^{0}=$ Quasiconservative source.

$\vec{S}^{1}=$ Conservative source.

$\vec{S}^{2}=$ No resistance quasiconservative source.

$S_{f}=$ Resistance slope.

$\vec{S}^{f}=$ Resistance vector.

$S_{x}=$ Bed slope: $S_{x}=-\tan \theta$.

$S P_{i+1 / 2}=$ Surplus of numerical flux between $i$-th and $i+1$-th cells. 
$t=$ Time.

$t_{\text {decay }}=$ Decay time for the 'invariant depth test'.

$T_{f}=$ Resistance corrector term.

$U=$ Velocity of the discontinuity step in the 'DNF test'.

$\vec{U}=$ Conserved variables vector.

$\vec{U}^{C}=$ Corrected value of $\vec{U}$ at $n+1$-th time step (i.e., $\vec{U}^{C}=\vec{U}^{P}+\Delta \vec{U}^{C}$ ).

$\vec{U}^{P}=$ Predicted value of $\vec{U}$ at $n+1$-th time step (i.e., $\vec{U}^{P}=\vec{U}^{n}+\Delta \vec{U}^{P}$ ).

$u=\operatorname{Velocity}(x$ component $)$.

$u^{\prime}=\operatorname{Velocity}\left(x^{\prime}\right.$ component)

$\vec{v}=$ Vector velocity.

$w=\operatorname{Velocity}(z$ component $)$

$w^{\prime}=\operatorname{Velocity}\left(z^{\prime}\right.$ component $)$.

$x=$ Spatial coordinate $\perp \vec{g}$.

$x^{\prime}=$ Spatial coordinate in primed coordinate system $\left(x^{\prime} \| z_{b}\right.$ surface $)$.

$z=$ Spatial coordinate $\| \vec{g}$.

$z^{\prime}=$ Spatial coordinate in primed coordinate system $\left(z^{\prime} \perp z_{b}\right.$ surface $)$.

$z_{b}=$ Bed level.

$z_{s}=$ Surface level.

\section{REFERENCES}


Beguería, S., van Asch, T. J., Malet, J. P., and Gröndahl, S. (2009a). "A GIS-based numerical model for simulating the kinematics of mud and debris flows over complex terrain." Natural Hazards in Earth System Sciences, 9, 1897-1909.

Beguería, S., van Hees, M. J., and Geertsema, M. (2009b). Landslide processes: from geomorphology mapping to dynamic modelling. CERG Editions, Strasbourg, Chapter Comparison of three landslide runout models on the Turnoff Creek rock avalanche, British Columbia, 243-247.

Bertolo, P. and Wieczorek, G. F. (2005). "Calibration of numerical models for small debris flows in yosemite valley, california, usa." Natural Hazards in Earth System Sciences, 5, 993-1001.

Brufau, P., García-Navarro, P., Ghilardi, P., Natale, L., and Savi, F. (2000). "1D mathematical modelling of debris flow." Journal of Hydraulic Research, 38(6), 435-446.

Burguete, J. (2003). "Modelos unidimensionales de flujos de superficie libre y transporte en geometrías irregulares: aplicación al flujo en ríos." Ph.D. thesis, University of Zaragoza, Zaragoza, Spain.

Burguete, J. and García-Navarro, P. (2001). "Efficient construction of high-resolution TVD conservative schemes for equations with source terms: application to shallow water flows." International Journal for Numerical Methods in Fluids, 37(2), 209-248.

Burguete, J. and García-Navarro, P. (2004). "Improving simple explicit methods for unsteady open channel and river flow." International Journal for Numerical Methods in Fluids, $45(2), 125-156$.

Burguete, J., García-Navarro, P., and Murillo, J. (2006). "Numerical boundary conditions for globally mass conservative methods to solve the shallow-water equations and applied to river flow." International Journal for Numerical Methods in Fluids, 51(6), 585-615.

Burguete, J., García-Navarro, P., and Murillo, J. (2008). "Friction term discretization and limitation to preserve stability and conservation in the 1D shallow-water model: application to unsteady irrigation and river flow." International Journal for Numerical Methods 
in Fluids, 58(4), 403-425.

Burguete, J., García-Navarro, P., Murillo, J., and García-Palacín, I. (2007). "Analysis of the friction term in the one-dimensional shallow water model." ASCE Journal of Hydraulic Engineering, 133(9), 1048-1063.

Courant, R., Friedrichs, K. O., and Lewy, H. (1928). "Über die partiellen differenzengleichungen der mathematischen Physik." Math. Ann., 100, 32-74.

Coussot, P. (1997). Mudflow rheology and dynamics. IAHR /AA Balkema, Rotterdam, The Nederlands.

Coussot, P. and Ancey, C. (1999). "Rheophysical classification of concentrated suspensions and granular pastes." Phys. Rev. E, 59, 4445-4457.

de Saint-Venant, A. J. C. B. (1871). "Théorie de mouvement non-permanent des eaux avec application aux crues de rivières et á l'introduction de marées dans leur lit.

Delis, A. I., Nikolos, I. K., and Kazolea, M. (2011). "Performance and comparison of cellcentered and node-centered unstructured finite volume discretizations for shallow water free surface flows." Archives of Computational Methods in Engineering, 18(1), 57-118.

Denlinger, R. P. and Iverson, R. M. (2001). "Flow of variably fluidized granular masses across three-dimensional terrain." Journal of Geophysical Research, 106, 553-566.

Hungr, O. (1995). "A model for the runout analysis of rapid flow slide, debris flow, and avalanches." Canadian Geotechnical Journal, 32, 610-623.

Hungr, O. and Evans, S. G. (1996). "Rock avalanche runout prediction using a dynamic model." 7th International Symposium on Landslides, 233-238.

Iverson, R. M. (1997). "The physics of debris flows." Reviews of Geophysics, 35(3), 245-296.

Kent, A. and Hungr, O. (1995). "Runout characteristics of debris from dump failures in mountainous terrain: stage 2: analysis, modelling and prediction.

Koerner, H. J. (1976). "Reichweite und geschwindigkeit von bergstürzen und fleisschneelawinen." Rock Mechanics, 8, 225-256.

Laigle, D. and Coussot, P. (1997). "Numerical modelling of mudflows." ASCE Journal of 
Hydraulic Engineering, 123(7), 617-623.

Landau, L. and Lifchitz, E. (1988). Fluid Mechanics (2nd edition). Pergamon Press, Oxford. MacDonald, I., Baines, M. J., Nichols, N. K., and Samuels, P. G. (1997). "Analytical benchmark solutions for open-channel flows." ASCE Journal of Hydraulic Engineering, 123(11), $1041-1045$.

Mangeney-Castelnau, A., Bouchut, F., Vilotte, J. P., Lajeunesse, E., Aubertin, A., and Pirulli, M. (2005). "On the use of Saint Venant equations to simulate the spreading of a granular mass." Journal of Geophysical Research, 110, B09103.

McLellan, P. J. and Kaiser, P. K. (1984). "Application of a two-parameter model to rock avalanches in the mackenzine mountains." 4th International Symposium on Landslides, $135-140$.

Murillo, J. and García-Navarro, P. (2012). "Wave Riemann description of friction terms in unsteady shallow flows: Application to water and mud/debris floods." Journal of Computational Physics, 231, 1963-2001.

Murillo, J., García-Navarro, P., and Burguete, J. (2007). "The influence of source terms on stability, accuracy and conservation in 2D shallow flow simulation using triangular finite volumes." International Journal for Numerical Methods in Fluids, 54, 543-590.

Murillo, J., García-Navarro, P., and Burguete, J. (2009). "Time step restrictions for wellbalanced shallow water solutions in non-zero velocity steady states." International Journal for Numerical Methods in Fluids, 60(3), 1351-1377.

O’Brien, J. S. and Julien, P. Y. (1993). "Two-dimensional water flood and mudflow simulation." ASCE Journal of Hydraulic Engineering, 119(2), 244-261.

Rickenmann, D. and Koch, T. (1997). "Comparison of debris flow modelling approaches." First International Conference on Debris-Flow Hazards Mitigation: Mechanics, Prediction, and Assessment, ASCE, ed., New York, C. L. Chen, 576-585.

Roe, P. L. (1981). "Approximate Riemann solvers, parameter vectors, and difference schemes." Journal of Computational Physics, 43(2), 357-372. 
Takahashi, T. (1991). Debris flow. Balkema, Rotterdam.

Voellmy, A. (1955). Über die Zerstörungskraft von Lawinen. Schweizer. Bauzeitung. 
TABLE 1: Parameters for the subcritical and supercritical cases of the steady flow tests with analytical solutions.

\begin{tabular}{c|cc} 
& Subcritical case & Supercritical case \\
\hline$q\left(\mathrm{~m}^{2} / \mathrm{s}\right)$ & 5 & 5 \\
$\xi\left(\mathrm{s}^{2} / \mathrm{m}\right)$ & 0.003 & 0.003 \\
$\tan \varphi$ & 0.05 & 0.05 \\
$h_{0}(\mathrm{~m})$ & 2 & 1
\end{tabular}


TABLE 2: Ideal dambreak initial conditions and rheology parameters.

\begin{tabular}{c|cc} 
& Case I & Case II \\
\hline$h_{i}(\mathrm{~m})$ & 10 & 10 \\
$\tan \varphi$ & 0.7 & 0.5 \\
$\xi\left(\mathrm{s}^{2} / \mathrm{m}\right)$ & 0 & 0.003
\end{tabular}




\section{List of Figures}

1 Reference svstems on a well developed flow over strong constant slope. . . . 38

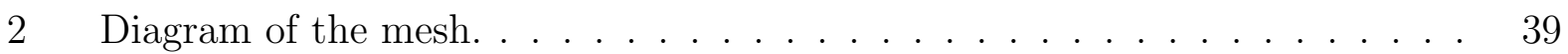

3 Numerical error due to unphvsical overestimation of the resistance. . . . . 40

4 Integration limits leading to the MREL method . . . . . . . . . . . . . 41

5 Analvtical soil and surface levels for the subcritical case. . . . . . . . . . . 42

6 Steadv flow test: PRT vs. URT in subcritical case. . . . . . . . . . . . . . 43

$7 \quad$ Analvtical soil and surface levels for the supercritical case. . . . . . . . . . . 44

8 Steadv flow test: PRT vs. URT in supercritical case. . . . . . . . . . . . 45

$9 \quad$ Ideal dambreak test I: general profiles . . . . . . . . . . . . . 46

$10 h$ profiles in ideal dambreak I with and without MREL protection at $t=10 \mathrm{~s}$. 47

$11 q$ profiles in ideal dambreak I with and without MREL protection at $t=10 \mathrm{~s} . \quad 48$

$12 q$ profiles in ideal dambreak II with and without DSC at $t=1$ s. . . . . . 49

$13 h$ profiles in ideal dambreak II with and without DCL protection at $t=1 \mathrm{~s}$. . $\quad 50$

14 a decav evolution in the invariant depth test . . . . . . . . . . . . . . . 51

15 Diagram of the DNF test . . . . . . . . . . . . . . 52

16 DNF test: initial and $t=25$ s profiles of $q \ldots \ldots \ldots$ 


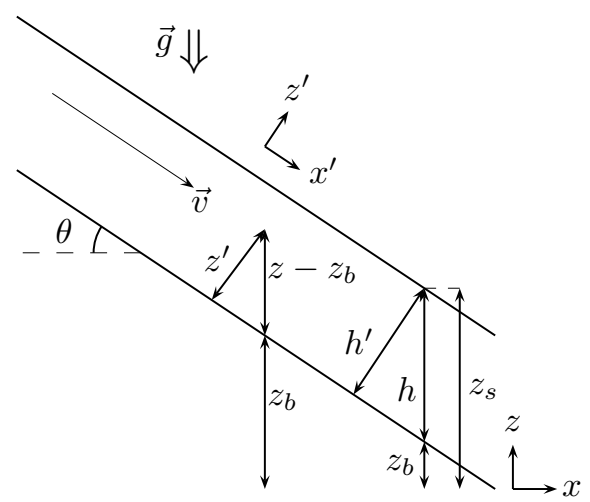

FIG. 1: Reference systems on a well developed flow over strong constant slope. The velocity in this case is parallel to the bed and the surface. Then, in primed frame $\vec{v}=\left(u^{\prime}, 0\right)$. Note also $h^{\prime}=h \cos \theta$. 


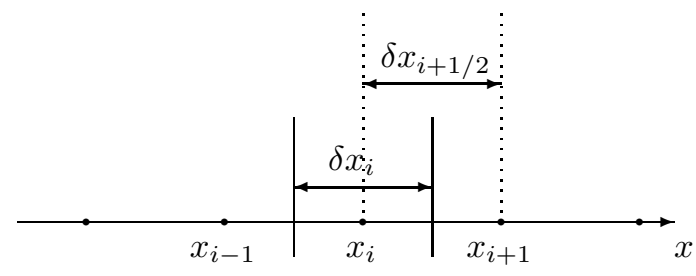

FIG. 2: Diagram of the mesh. 


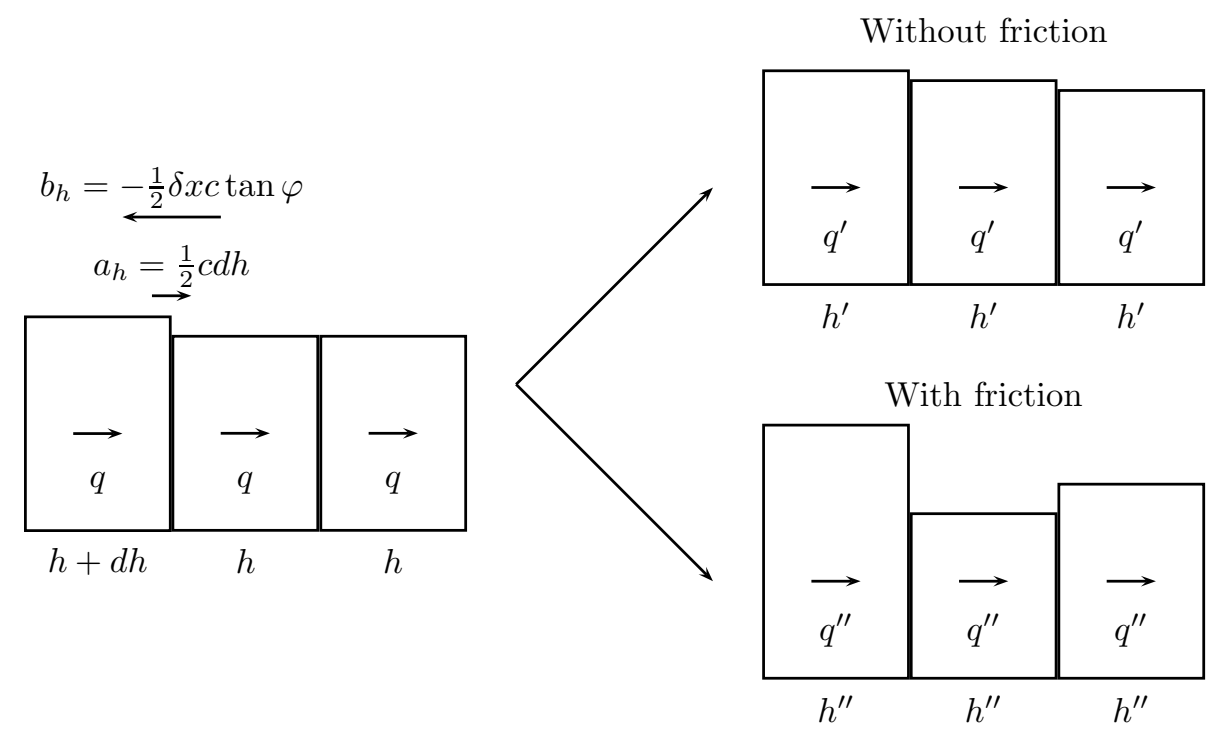

FIG. 3: Numerical error due to unphysical overestimation of the resistance for low values of $q, a_{h} b_{h}<0$ and $\left|a_{h}\right|<\left|b_{h}\right|$. 


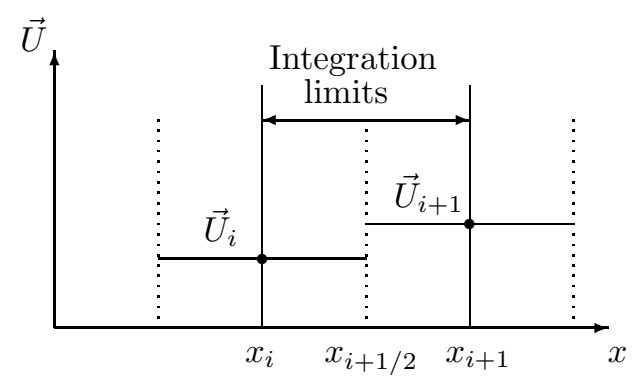

FIG. 4: Integration limits leading to the MREL method. 


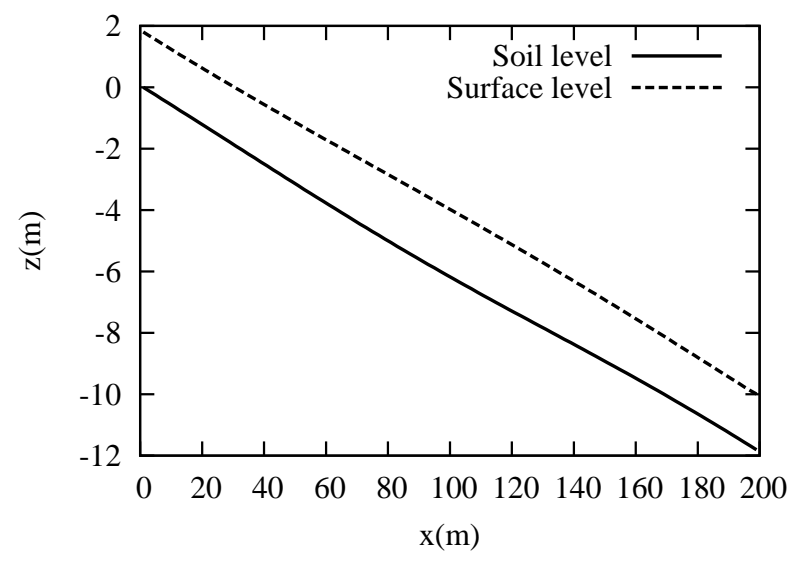

FIG. 5: Analytical soil and surface levels for the subcritical case. 

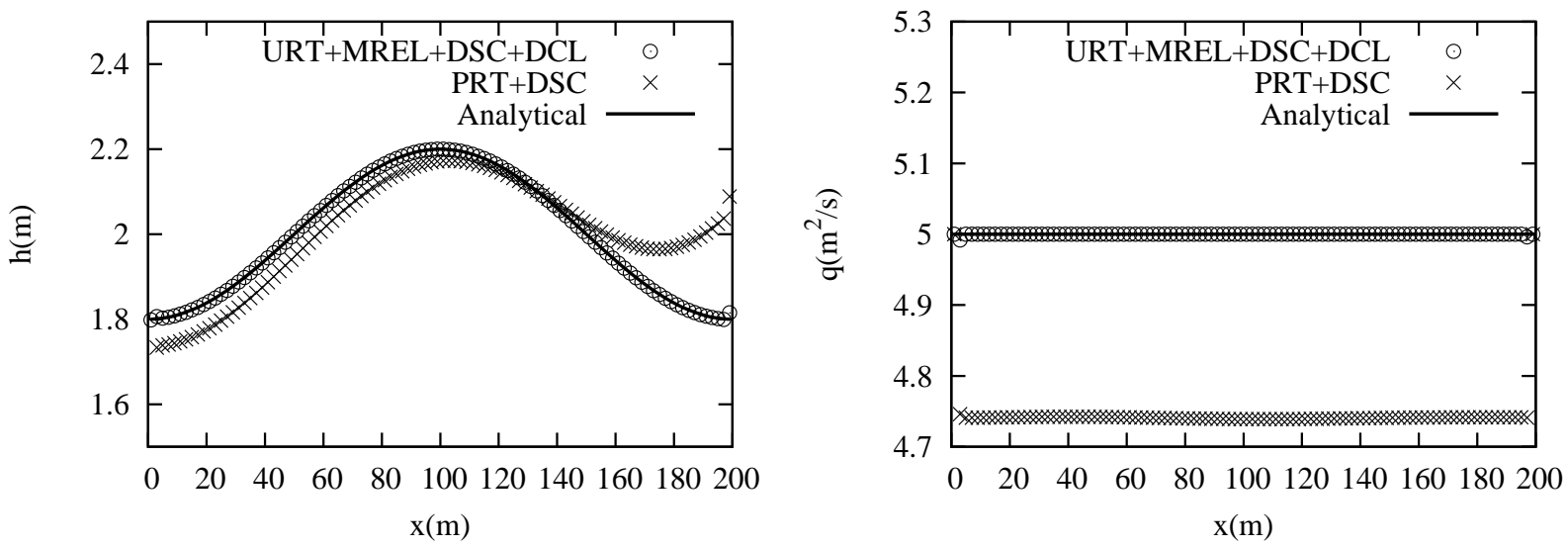

FIG. 6: Steady flow test: comparing PRT with unified method in the subcritical case. $h$ (left) and $q$ (right) profiles. 


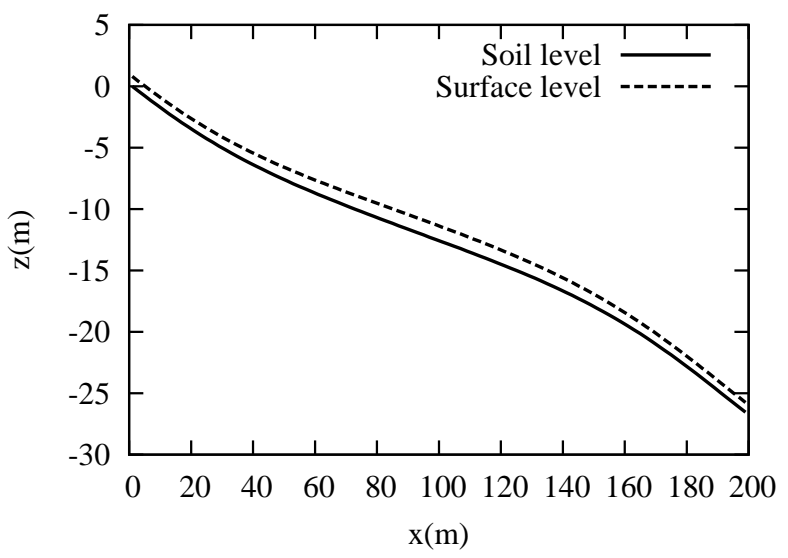

FIG. 7: Analytical soil and surface levels for the supercritical case. 

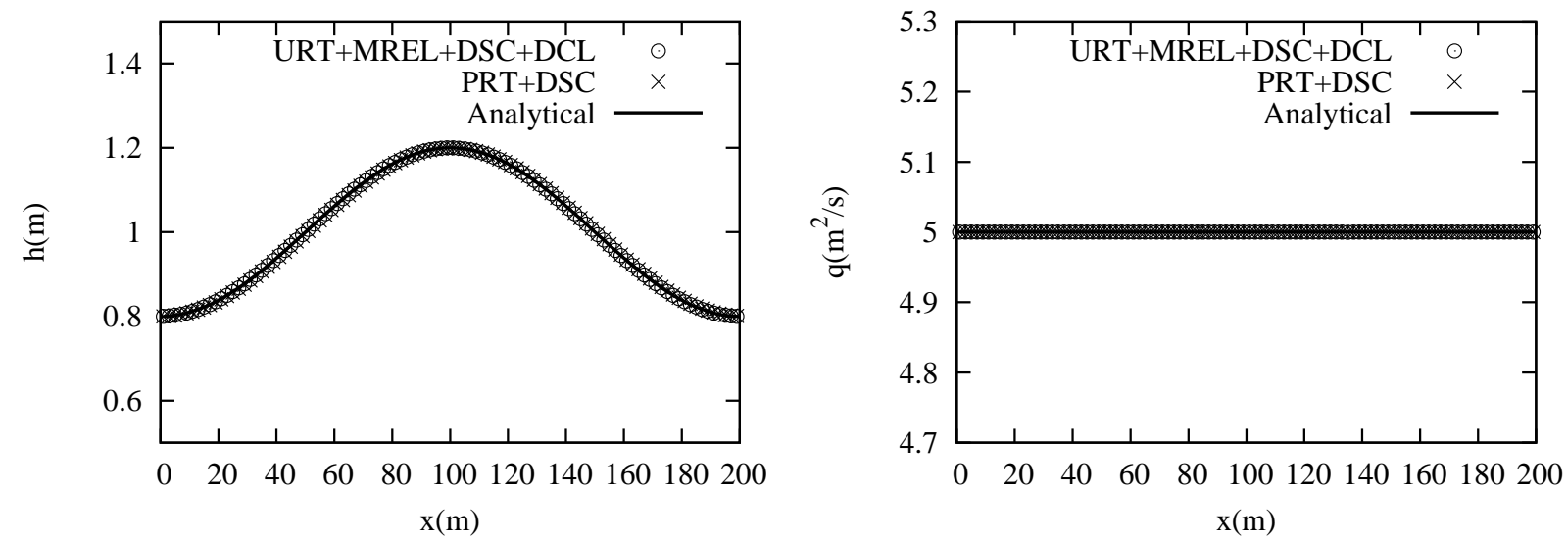

FIG. 8: Steady flow test: comparing PRT with unified method in the supercritical case. $h$ (left) and $q$ (right) profiles. 

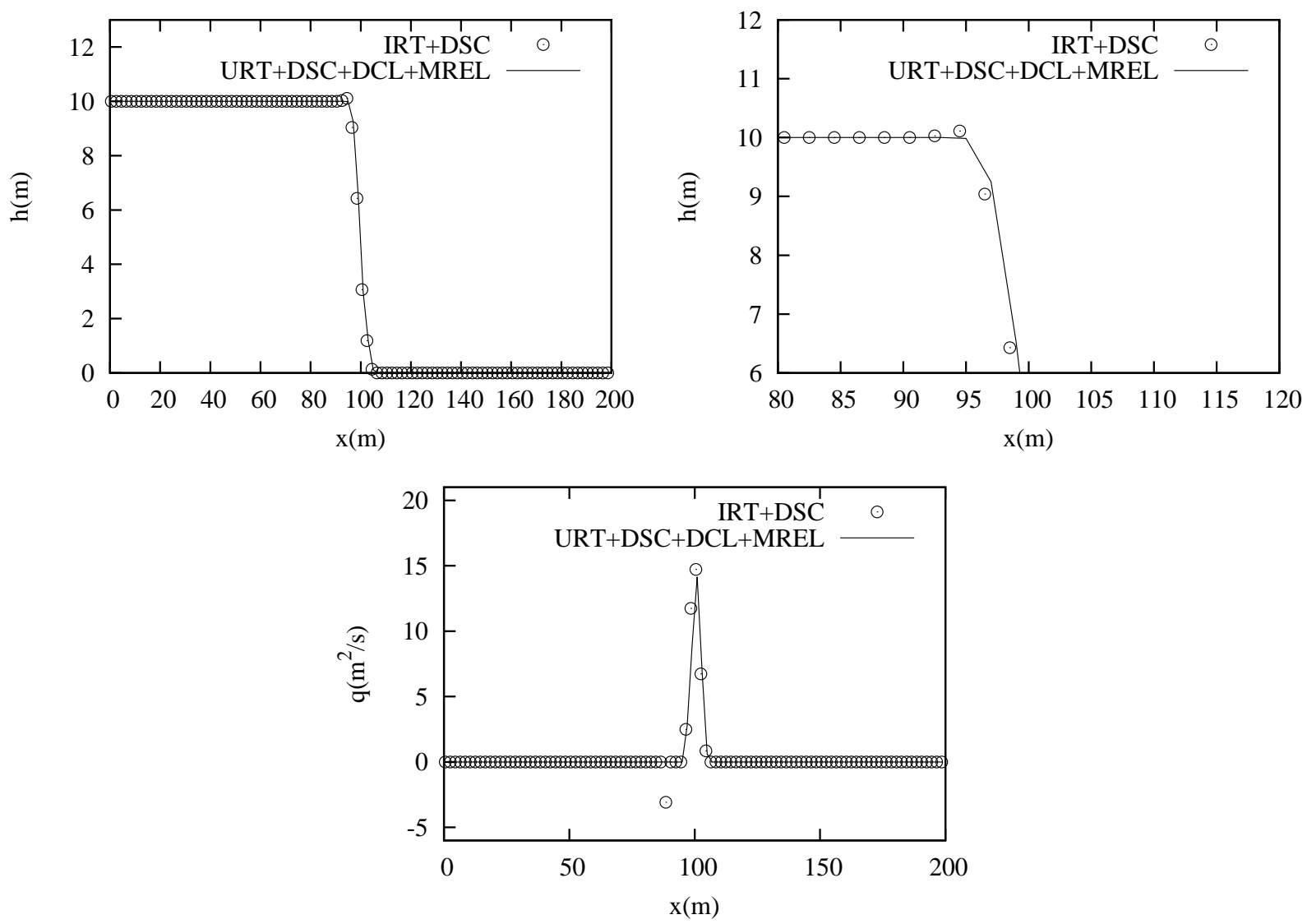

FIG. 9: Ideal dambreak test I: general profile of $h$ (top left) and detail (top right), and $q$ profile (bottom) with IRT and URT and $t=0.27 \mathrm{~s}$. 

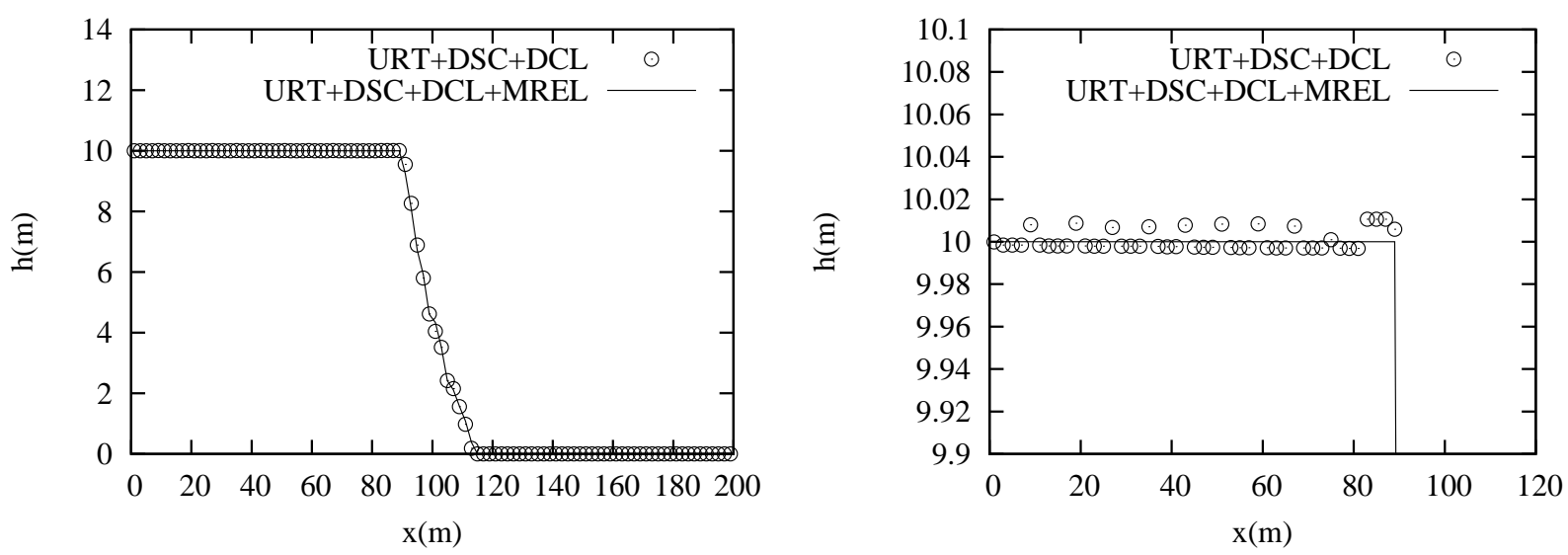

FIG. 10: $h$ profiles of ideal dambreak case I with MREL protection enabled and disabled at $t=10 \mathrm{~s}$ : general view (left) and detail (right). 


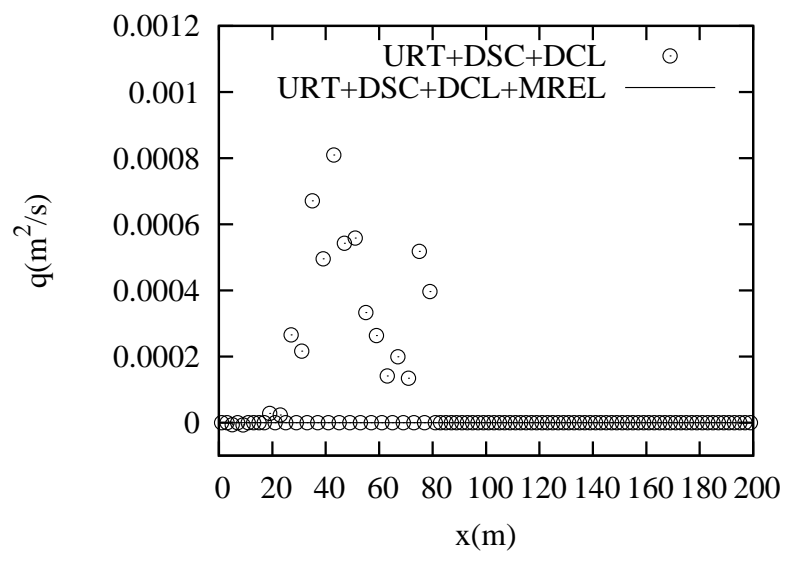

FIG. 11: $q$ profiles of ideal dambreak case I with MREL protection enabled and disabled at $t=10 \mathrm{~s}$. 

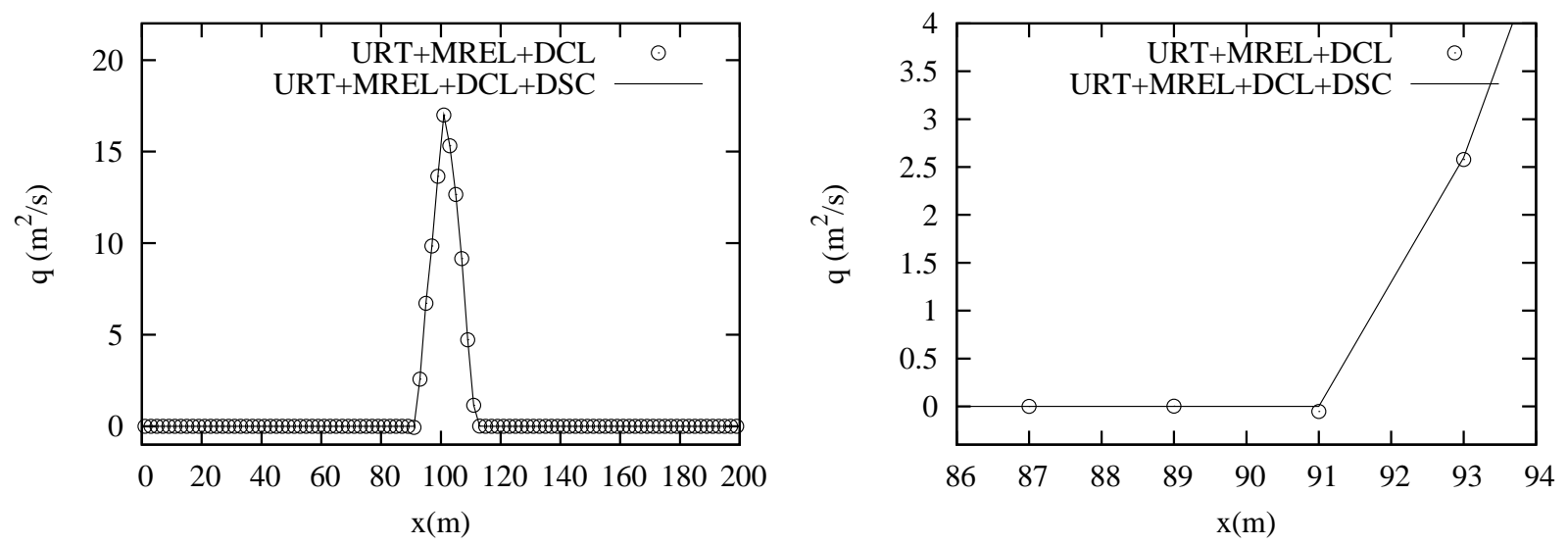

FIG. 12: $q$ profiles of ideal dambreak case II with DSC enabled and disabled at $t=1 \mathrm{~s}$ : general view (left) and detail (right). 

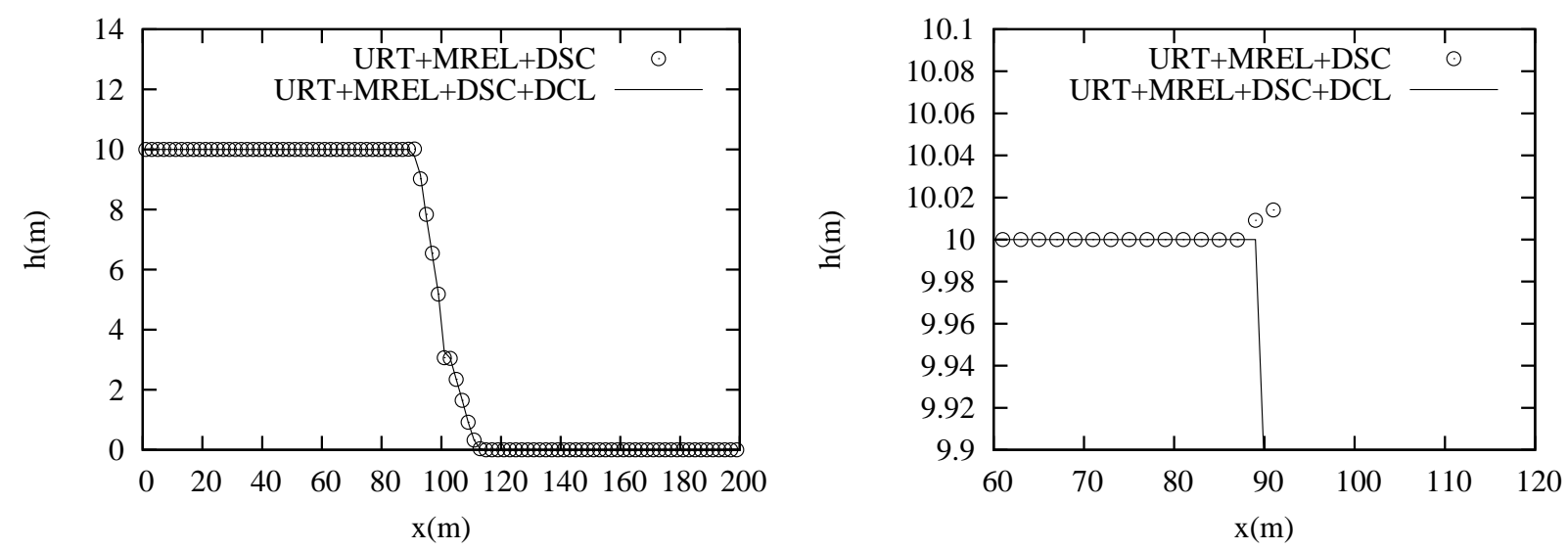

FIG. 13: $h$ profiles of ideal dambreak case II with DCL correction enabled and disabled at $t=1 \mathrm{~s}$. General view (left) and detail (right). 

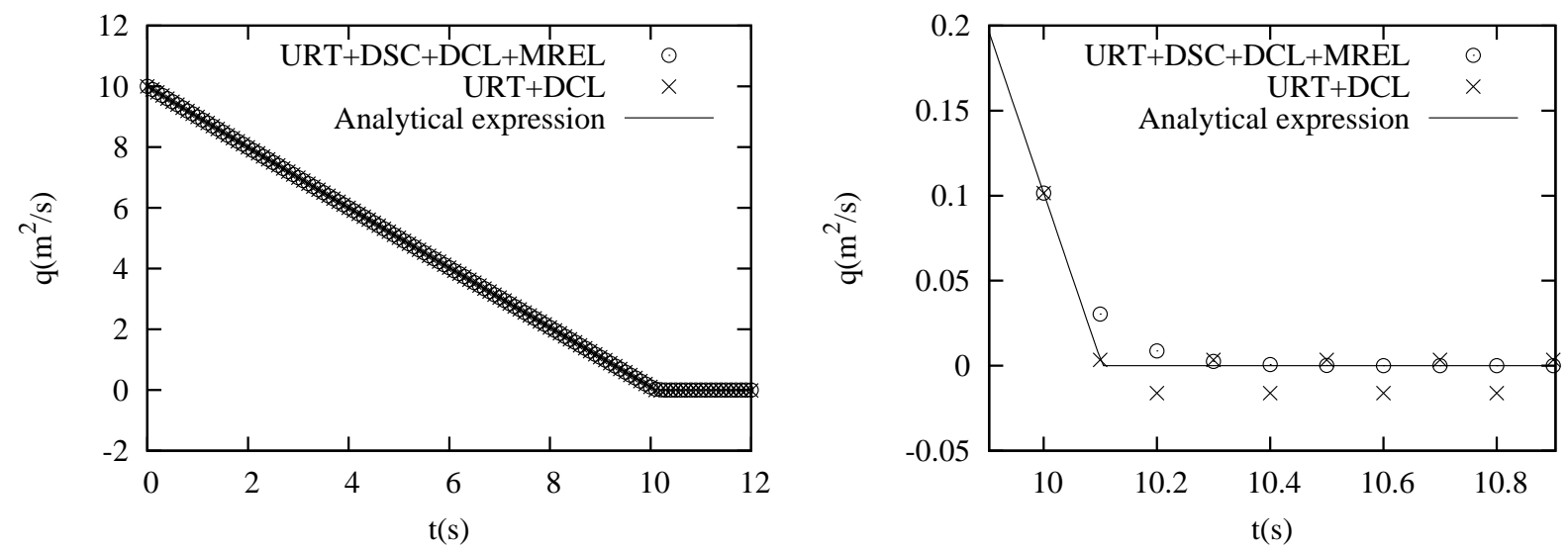

FIG. 14: $q$ decay evolution in the invariant depth test. General view (left) and detail (right). 


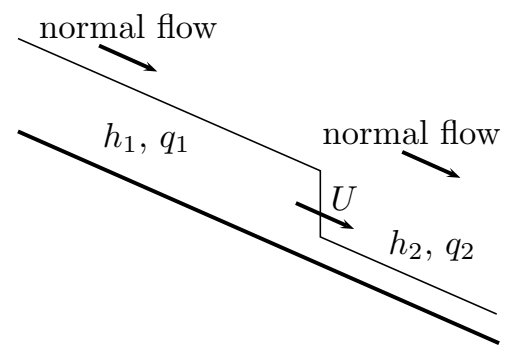

FIG. 15: Diagram of the DNF test. 


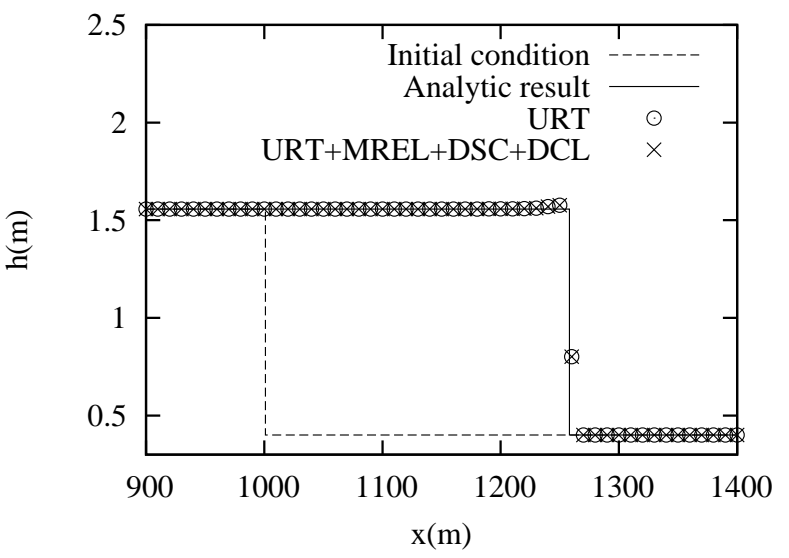

FIG. 16: DNF test: initial and $t=25$ s profiles of $q$. 